\title{
The Digital Brain Bank, an open access platform for post-mortem datasets
}

Benjamin C. Tendler ${ }^{1}$, Taylor Hanayik ${ }^{1}$, Olaf Ansorge ${ }^{2}$, Sarah Bangerter-Christensen ${ }^{2}$, Gregory S. Berns ${ }^{3}$, Mads F. Bertelsen ${ }^{4}$, Katherine L. Bryant ${ }^{1}$, Sean Foxley ${ }^{1,5}$, Martijn P. van den Heuvel ${ }^{6,7}$, Amy F.D. Howard ${ }^{1}$, Istvan Huszar ${ }^{1}$, Alexandre A. Khrapitchev ${ }^{8}$, Anna Leonte ${ }^{2}$, Paul R. Manger ${ }^{9}$, Ricarda A.L. Menke ${ }^{1}$, Jeroen Mollink ${ }^{1}$, Duncan Mortimer ${ }^{1}$, Menuka Pallebage-Gamarallage ${ }^{2}$, Lea Roumazeilles ${ }^{10}$, Jerome Sallet ${ }^{10,11}$, Lianne H. Scholtens ${ }^{6}$, Connor Scott ${ }^{2}$, Adele Smart ${ }^{1,2}$, Martin R. Turner ${ }^{1,2}$, Chaoyue Wang $^{1}$, Saad Jbabdi ${ }^{*}$, Rogier B. Mars ${ }^{1,12^{*}}$, Karla L. Miller ${ }^{1 *}$

${ }^{1}$ Wellcome Centre for Integrative Neuroimaging, FMRIB, Nuffield Department of Clinical Neurosciences, University of Oxford, United Kingdom

${ }^{2}$ Division of Clinical Neurology, Nuffield Department of Clinical Neurosciences, University of Oxford, United Kingdom

${ }^{3}$ Psychology Department, Emory University, Atlanta, Georgia, United States of America

${ }^{4}$ Centre for Zoo and Wild Animal Health, Copenhagen Zoo, Frederiksberg, Denmark

${ }^{5}$ Department of Radiology, University of Chicago, Chicago, Illinois, United States of America

${ }^{6}$ Department of Complex Trait Genetics, Centre for Neurogenomics and Cognitive Research, Amsterdam Neuroscience, Vrije Universiteit Amsterdam, Amsterdam, The Netherlands

7 Department of Child Psychiatry, Amsterdam Neuroscience, Amsterdam UMC, Vrije Universiteit Amsterdam, Amsterdam, The Netherlands

${ }^{8}$ Medical Research Council Oxford Institute for Radiation Oncology, University of Oxford, Oxford, United Kingdom

${ }^{9}$ School of Anatomical Sciences, Faculty of Health Sciences, University of the Witwatersrand, Johannesburg, South Africa

${ }^{10}$ Wellcome Centre for Integrative Neuroimaging, Department of Experimental Psychology, University of Oxford, Oxford, United Kingdom

${ }^{11}$ Stem Cell and Brain Research Institute, Université Lyon 1, INSERM, Bron, France

${ }^{12}$ Donders Institute for Brain, Cognition and Behaviour, Radboud University Nijmegen, Nijmegen, The Netherlands

*Authors provided equal contribution 
bioRxiv preprint doi: https://doi.org/10.1101/2021.06.21.449154; this version posted September 1, 2021. The copyright holder for this preprint (which was not certified by peer review) is the author/funder, who has granted bioRxiv a license to display the preprint in perpetuity. It is made available under aCC-BY 4.0 International license.

\begin{abstract}
Post-mortem MRI provides the opportunity to acquire high-resolution datasets to investigate neuroanatomy, and validate the origins of image contrast through microscopy comparisons. We introduce the Digital Brain Bank (open.win.ox.ac.uk/DigitalBrainBank), an interactive data discovery and release platform providing open access to curated, multimodal post-mortem neuroimaging datasets. Datasets span three themes - Digital Neuroanatomist: datasets for detailed neuroanatomical investigations; Digital Brain Zoo: datasets for comparative neuroanatomy; Digital Pathologist: datasets for neuropathology investigations. The first Digital Brain Bank release includes twenty one distinctive whole-brain diffusion MRI datasets for structural connectivity investigations, alongside microscopy and complementary MRI modalities. This includes one of the highest-resolution whole-brain human diffusion MRI datasets ever acquired, whole-brain diffusion MRI in fourteen non-human primate species, and one of the largest post-mortem whole-brain cohort imaging studies in neurodegeneration. Taken together, the Digital Brain Bank provides a cross-scale, cross-species investigation framework facilitating the incorporation of post-mortem data into neuroimaging studies.
\end{abstract}




\section{Introduction}

Magnetic resonance imaging (MRI) occupies a unique position in the neuroscience toolkit. In humans, MRI is used at the single subject level diagnostically, and is increasingly deployed at the population level in epidemiology (Marcus et al., 2007; Miller et al., 2016; Snoek et al., 2021; Van Essen et al., 2013). MRI is wellestablished in the context of imaging causal manipulations in experimental organisms ranging from mice (Denic et al., 2011; Thiessen et al., 2013) to non-human primates (Absinta et al., 2017; Klink et al., 2021), and provides precise measurements in cellular and tissue preparations (Wilhelm et al., 2012). This extensive landscape of overlap with the broader neuroscience toolkit creates the potential for MRI to facilitate integration between technologies and investigations. Although MRI hardware and acquisition protocols often need to be tailored to a specific domain, the signals measured in all these settings are fundamentally the same (Boon et al., 2019). There are few methods available to neuroscientists that span this breadth of domains.

One challenge to the use of MRI as a bridging technology is the need for common measurements - for example, the same MRI measurements made across multiple species, or MRI and microscopy measurements in the same brain tissue (Mars et al., 2021). Post-mortem MRI provides unique opportunities for such common measurements. MRI in post-mortem tissue can be used to identify the origins of image contrast through integration with microscopy (Keren et al., 2015; Langkammer et al., 2012; Mollink et al., 2017), directly addressing concerns over the non-specificity of MRI signals. In this context, post-mortem MRI data are important because they share common signal forming mechanisms with in vivo MRI and a common tissue state with microscopy, providing a framework for investigation across multiple spatial scales. Post-mortem MRI facilitates comparative anatomy investigations in species that are not traditionally accessible for in-vivo imaging (Berns et al., 2015; Bhagwandin et al., 2017; Grewal et al., 2020; Heuer et al., 2019), including extinct species (Berns \& Ashwell, 2017). Long post-mortem scans provide the opportunity to push the boundaries of spatial resolution, providing whole human brain coverage reaching voxel sizes of $100-500 \mu \mathrm{m}$ (Edlow et al., 2019; Foxley et al., 2016; Fritz et al., 2019; Weigel et al., 2021), edging closer to microscopy techniques but benefitting from compatibility with in-vivo imaging. As a non-destructive technique, post-mortem MRI enables the examination of tissue microstructure whilst preserving tissue, facilitating repeat MRI measurements with novel contrasts and technologies, and more generally its integration with tools for post-mortem investigations (e.g. histopathology or proteomics).

In this work, we introduce the Digital Brain Bank (open.win.ox.ac.uk/DigitalBrainBank), a post-mortem data discovery and release online platform resulting from a decade of post-mortem MRI research at the University of Oxford. The Digital Brain Bank provides open access to a number of post-mortem neuroimaging datasets spanning investigations into human neuroanatomy, cross species neuroanatomy, and neuropathology. All datasets provide post-mortem MRI, including diffusion MRI, with complementary microscopy data (e.g. immunohistochemistry or polarised light imaging) included with some datasets.

Our post-mortem imaging research has been specifically aimed at achieving whole-brain post-mortem MRI to support investigation of multiple brain systems/regions and long-range connections (Foxley et al., 2014; Miller et al., 2011, 2012), and the first release to the Digital Brain Bank contains twenty one distinct whole brain post-mortem MRI datasets, including from whole human brains. All datasets are available to download, and prospective users of the Digital Brain Bank can explore a subset of data directly on the Digital Brain Bank website using Tview: a bespoke, open-source, web-based image viewer. Tview has been developed for efficient browsing of imaging data spanning drastically different spatial scales, from sub-micron resolution microscopy to millimetre MRI acquisitions. It enables real-time visualisation and interaction (zooming/panning) of both MRI and microscopy images, and with flexible overlays of different modalities.

The first release to the Digital Brain Bank includes data from multiple published projects covering a breadth of neuroimaging research, including whole-brain diffusion MRI in fourteen non-human primate species (Bryant et al., 2021; Roumazeilles et al., 2020, 2021), and one of the largest post-mortem whole-brain cohort imaging studies combining whole-brain MRI and microscopy in human neurodegeneration (Pallebage-Gamarallage et al., 2018). In addition, we present a previously unpublished project providing one of the highest-resolution whole-brain human diffusion MRI datasets ever acquired (500 $\mu \mathrm{m}$ isotropic resolution). The Digital Brain Bank will continue to grow over the coming years, with a number of further datasets already at the early stages of curation (A. Howard et al., 2019; Martins-Bach et al., 2021; Wu et al., 2021). 


\begin{tabular}{|c|c|c|c|c|}
\hline Category & Name & Contents: MRI & Contents: Microscopy & Relevant Publications \\
\hline $\begin{array}{l}\text { Digital } \\
\text { Anatomist }\end{array}$ & $\begin{array}{l}\text { Human High- } \\
\text { Resolution } \\
\text { Diffusion MRI-PLI }\end{array}$ & $\begin{array}{l}\text { Whole brain diffusion MRI }(500 \mu \mathrm{m}, 1 \text { and } 2 \mathrm{~mm} \text { iso.), } \\
\text { structural MRI }\left(312.5 \times 312.5 \times 500 \mu \mathrm{m}^{3}\right) \text {, quantitative T1 } \\
\left(0.75 \times 0.75 \times 1.6 \mathrm{~mm}^{3}\right) \text { and } \mathrm{T} 2\left(0.75 \times 0.75 \times 1.6 \mathrm{~mm}^{3}\right) \\
\text { maps: } \\
\text { - Control human brain: } 1 \mathrm{x}\end{array}$ & $\begin{array}{l}\text { Polarised light imaging ( } 4 \mu \mathrm{m} \text { in-plane) in the anterior commissure, } \\
\text { corpus callosum, pons, thalamus and visual cortex (same brain) }\end{array}$ & $\begin{array}{l}\text { Dataset described in this publication } \\
\text { (Methodology in Supporting Information), } \\
\text { Diffusion MRI processing described in } \\
\text { (Tendler et al., 2020), T2 mapping described } \\
\text { in (Tendler, Qi, et al., 2020) }\end{array}$ \\
\hline $\begin{array}{l}\text { Digital } \\
\text { Anatomist }\end{array}$ & $\begin{array}{l}\text { Human Callosum } \\
\text { MRI-PLI- } \\
\text { Histology }\end{array}$ & $\begin{array}{l}\text { Corpus callosum diffusion MRI ( } 400 \mu \mathrm{m} \text { iso.): } \\
\text { - Excised control human corpus callosum samples: } 3 x\end{array}$ & $\begin{array}{l}\text { Polarised light imaging ( } 4 \mu \mathrm{m} \text { in-plane), bright field microscopy } \\
\text { images of immunohistochemistry stains ( } 0.25 \mu \mathrm{m} \text { in-plane) for PLP } \\
\text { (myelin) and GFAP (astrocyte) in three excised human corpus } \\
\text { callosum samples (same samples) }\end{array}$ & (Mollink et al., 2017) \\
\hline $\begin{array}{l}\text { Digital } \\
\text { Brain Zoo }\end{array}$ & $\begin{array}{l}\text { Non-Human } \\
\text { Primates }\end{array}$ & $\begin{array}{l}\text { Whole brain diffusion MRI and structural MRI (selected } \\
\text { brains): } \\
\text { - Bushbaby (Galago senegalensis): } 1 \mathrm{x} \\
\text { - Capuchin monkey (Sepajus apella): } 1 \mathrm{x} \\
\text { - Chimpanzee (Pan troglodytes): } 2 \mathrm{x} \\
\text { - Colobus monkey (Colobus guereza): } 1 \mathrm{x} \\
\text { - Cotton-Top tamarin (Saguinus oedipus): } 1 \mathrm{x} \\
\text { - Golden Lion tamarin (Leontopithecus rosalia): } 1 \mathrm{x} \\
\text { - Hamadryas baboon (Papio hamadryas): } 1 \mathrm{x} \\
\text { - Macaque monkey (Macaca mulatta): } 3 \mathrm{x} \\
\text { - Mangabey (Lophocebus albigena): } 1 \mathrm{x} \\
\text { - Night monkey, (Aotus lemurinus): } 1 \mathrm{x} \\
\text { - Ring-tailed lemur (Lemur catta): } 3 \mathrm{x} \\
\text { - Saki monkey (Pithecia pithecia): } 1 \mathrm{x} \\
\text { - Western Lowland gorilla (Gorilla gorilla): } 1 \mathrm{x} \\
\text { - Woolly monkey (Lagothrix lagotricha): } 1 \mathrm{x}\end{array}$ & None & $\begin{array}{l}\text { 1x Western Lowland gorilla and } 1 x \\
\text { Chimpanzee described in (Roumazeilles et } \\
\text { al., 2020), 3x Macaque monkey and } 3 x \text { Ring- } \\
\text { Tailed Lemur described in (Roumazeilles et } \\
\text { al., 2021). Hamadryas baboon, Cotton-Top } \\
\text { tamarin and Golden Lion tamarin datasets } \\
\text { described in this publication (Methodology in } \\
\text { Supporting Information). All other datasets } \\
\text { described in (Bryant et al., 2021) }\end{array}$ \\
\hline $\begin{array}{l}\text { Digital } \\
\text { Brain Zoo }\end{array}$ & Marsupials & $\begin{array}{l}\text { Whole brain diffusion MRI and structural MRI: } \\
\text { - Tasmanian devil (Sarcophilus harrisii): } 2 x \\
\text { - Thylacine (Thylacinus cynocephalus): } 2 x\end{array}$ & None & (Berns \& Ashwell, 2017) \\
\hline $\begin{array}{l}\text { Digital } \\
\text { Brain Zoo }\end{array}$ & Cetaceans & $\begin{array}{l}\text { Whole brain diffusion MRI (1.3 mm iso.) and structural } \\
\text { MRI }\left(640 \times 640 \times 500 \mu \mathrm{m}^{3}\right) \\
\text { - Common dolphin (Delphinus delphis): } 1 \times \\
\text { - Pantropical dolphin (Stenella attenuata): } 1 \times\end{array}$ & None & (Berns et al., 2015) \\
\hline $\begin{array}{l}\text { Digital } \\
\text { Brain Zoo }\end{array}$ & Carnivora & $\begin{array}{l}\text { Whole brain diffusion MRI ( } 600 \mu \mathrm{m} \text { iso.) and structural } \\
\text { MRI ( } 220 \mu \mathrm{m} \text { iso.): } \\
\text { - European wolf (Canis lupus): } 1 \mathrm{x}\end{array}$ & None & $\begin{array}{l}\text { Dataset described in this publication } \\
\text { (Methodology in Supporting Information) }\end{array}$ \\
\hline $\begin{array}{l}\text { Digital } \\
\text { Pathologist }\end{array}$ & $\begin{array}{l}\text { Human ALS MRI- } \\
\text { Histology }\end{array}$ & $\begin{array}{l}\text { Whole brain diffusion MRI ( } 850 \mu \mathrm{m} \text { iso.), structural MRI, } \\
\text { quantitative T1, T2 and T2* maps (selected brains), } \\
\text { magnetic susceptibility maps (selected brains): } \\
\text { - Amyotrophic lateral sclerosis (ALS) human brains: } 12 x \\
\text { - Control human brains: } 3 x\end{array}$ & $\begin{array}{l}\text { Bright field microscopy images of immunohistochemistry stains } \\
\text { (0.50 } \mu \mathrm{m} \text { in-plane, exception pTDP43: } 0.25 \mu \mathrm{m} \text { in-plane) for pTDP- } \\
43, \text { IBA1 (pan microglia), CD68 (activated microglia/macrophages), } \\
\text { PLP (myelin), SMI-312 (axonal phosphorylated neurofilaments), } \\
\text { and ferritin (iron storage, subset of regions) in the anterior } \\
\text { cingulate cortex, corpus callosum, hippocampus, primary motor } \\
\text { cortex and visual cortex (same brains). Selected histology available } \\
\text { in } 2 \text { brains in first data release (1x ALS and } 1 \times \text { Control - remaining } \\
\text { histology being actively curated). }\end{array}$ & $\begin{array}{l}\text { Project description provided in } \\
\text { (Pallebage-Gamarallage et al., 2018), } \\
\text { Magnetic susceptibility and T2* mapping } \\
\text { protocol described in (Wang et al., 2020), } \\
\text { Diffusion MRI processing described in } \\
\text { (Tendler et al., 2020), T2 mapping described } \\
\text { in (Tendler, Qi, et al., 2020) }\end{array}$ \\
\hline
\end{tabular}

Table 1: Description of all datasets provided in the first release to the Digital Brain Bank. MRI resolutions provided when consistent across all images in a single dataset (iso. $=$ isotropic resolution). 


\section{Results}

The Digital Brain Bank is accessible at open.win.ox.ac.uk/DigitalBrainBank. Datasets have been organised into categories reflecting three predominant themes of post-mortem neuroimaging research:

- Digital Anatomist: Datasets for detailed neuroanatomical investigations.

- Digital Brain Zoo: Datasets for comparative neuroanatomy.

- Digital Pathologist: Datasets for neuropathology investigations.

Here we provide an overview of each theme, with examples from available datasets in the first release to the Digital Brain Bank. A brief description of all the datasets provided with the first release, alongside relevant publications, is provided in Table 1.

\section{Digital Anatomist}

Datasets within the Digital Anatomist provide a new direction for answering fundamental questions in neuroanatomy, through ultra-high resolution MRI data and complementary microscopy within the same sample.

The long scan times available in post-mortem MRI affords imaging at ultra-high spatial resolutions, facilitating the delineation of small tissue structures within the human brain, one of the key aims of the Digital Anatomist. Often, post-mortem investigations are limited to small sections of excised brain tissue that represent a limited anatomical region. However, our developments in whole-brain post-mortem diffusion imaging (Foxley et al., 2014; McNab et al., 2009; Miller et al., 2011, 2012; Tendler, Foxley, Hernandez-Fernandez, et al., 2020) provide the opportunity to investigate structural connectivity and gross neuroanatomy, at scales that are unobtainable in vivo. These developments have culminated in the Human High-Resolution Diffusion MRI-PLI dataset, providing one of the highest-resolution whole-brain human diffusion MRI datasets ever acquired (500 $\mu \mathrm{m}$ isotropic resolution), as shown in Fig. 1. Companion datasets acquired at $1 \mathrm{~mm}$ and $2 \mathrm{~mm}$ (isotropic) provide a comparison at cutting-edge and conventional in vivo resolutions (Fig. 1a).

In addition to providing a new insight into human neuroanatomy, these data can be used to inform experimental design and the interpretation of results. Here, the Human High-Resolution Diffusion MRI-PLI dataset enables users to identify the resolution required to visualise certain brain structures (Fig. 1a), and how spatial resolution impacts tractography performance (e.g. overcoming 'gyral bias' - Fig. 1b) (Cottaar et al., 2021; Schilling et al., 2018). Polarised light imaging (PLI) provides estimates of myelinated fibre orientation (Axer et al., 2011), and complementary PLI data acquired in a subset of brain regions ( $4 \mu \mathrm{m}$ in-plane) facilitates cross-scale comparisons (Fig. $1 \mathrm{~b}$ and c).

A further aim of the Digital Anatomist is to perform quantitative validations across modalities, relating MRI to microscopic measures. These kinds of analyses can only be achieved with accurately coregistered data, enabling pixel-wise comparisons across modalities acquired at drastically different spatial resolutions. This potential is most clearly seen in the Human Callosum MRI-PLI-Histology dataset, which provides diffusion MRI (400 $\mu \mathrm{m}$ isotropic), alongside complementary PLI ( $4 \mu \mathrm{m}$ in-plane) and histology (myelin and astrocytes) $(0.25$ $\mu \mathrm{m}$ in-plane) in three excised human corpus callosum samples (Mollink et al., 2017). These data offer multiple pathways of investigation, including the identification of the origins of image contrast; validation of microstructural models of tissue (Mollink et al., 2017); and developing unique models explicitly linking MRI with microscopy (A. F. Howard et al., 2019). Future datasets to be released to the Digital Anatomist will extend beyond human tissue for neuroanatomical investigations, such as the BigMac dataset, which will provide in vivo $\mathrm{MRI}$, post-mortem MRI, PLI and immunohistochemistry in a single, whole macaque brain (A. Howard et al., 2019). 


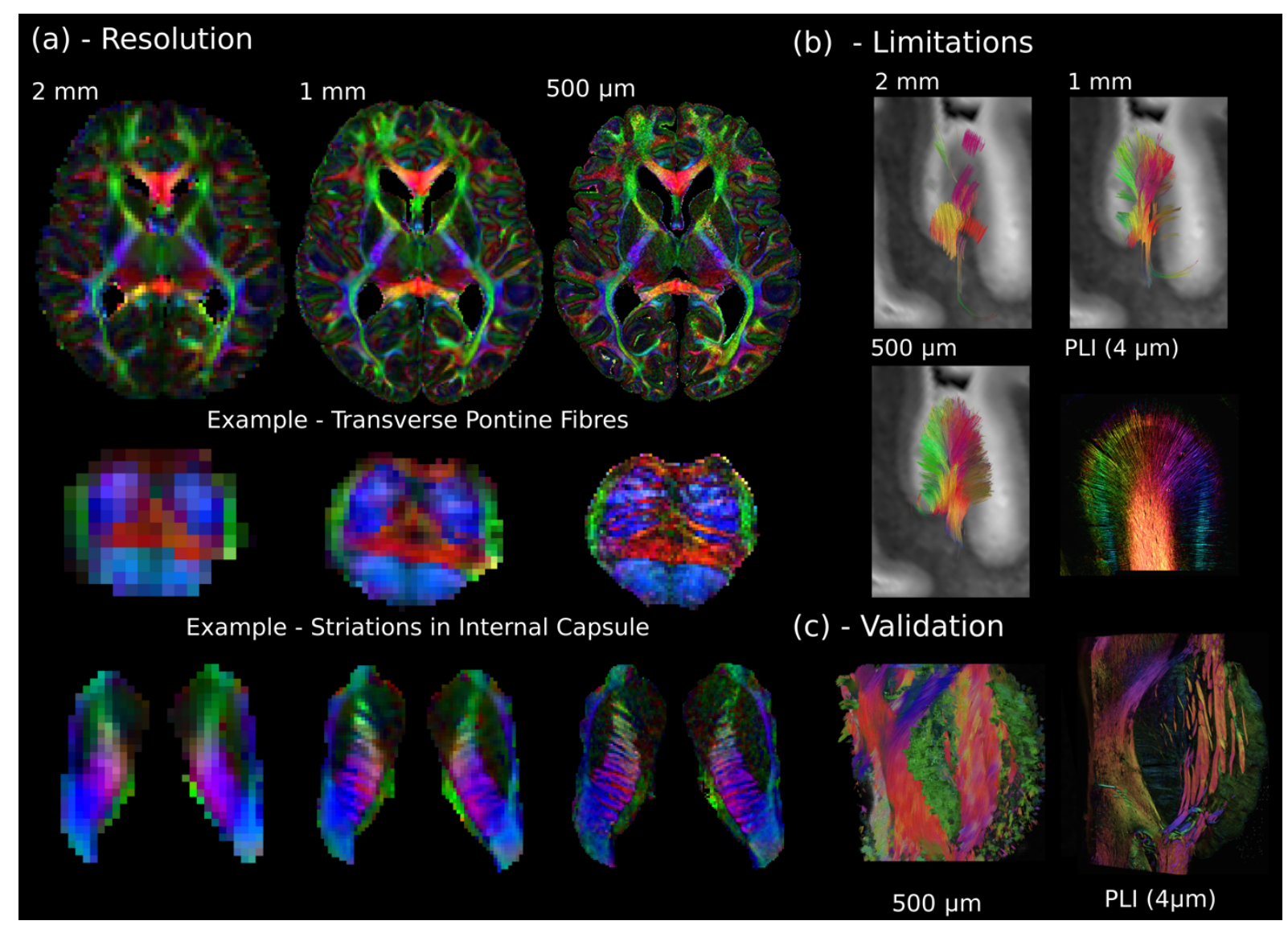

Figure 1: The Digital Anatomist. (a) Whole brain diffusion MRI data available in the Human High-Resolution Diffusion MRI$P L I$ dataset reveals the wealth of information provided at increased spatial scales, one of the key aims of the Digital Anatomist. Here, the $500 \mu \mathrm{m}$ dataset uncovers the information hidden at lower spatial resolutions, for example, visualising the interdigitating transverse pontine fibres with the corticospinal tract or striations through the internal capsule. (b) Similarly, datasets across multiple spatial scales can inform us of the limitations at reduced imaging resolutions. Here, gyral tractography (occipital lobe) reveals an overall pattern of fibres turning into the gyral bank at $0.5 \mathrm{~mm}$. At $1 \mathrm{~mm}$, an underestimation of connectivity at the gyral banks is observed, known as the 'gyral bias' (Cottaar et al., 2021; Schilling et al., 2018). At $2 \mathrm{~mm}$, tractography bears little resemblance to the expected architecture. Multi-modal comparisons enable us to validate our findings, with complementary Polarised Light Imaging (PLI) data at over two-orders of magnitude increase in resolution (125x) revealing a similar pattern of gyral connectivity, and (c) excellent visual agreement with tractography across the pons. (a) displays diffusion tensor principal diffusion direction maps (modulated by fractional anisotropy).

\section{Digital Brain Zoo}

\section{The Digital Brain Zoo provides curated datasets to investigate neuroanatomy in non-human species and} compare anatomy across species.

Post-mortem MRI has enormous potential to inform comparative neuroanatomy for three reasons. First, it enables scanning of species that would be extremely difficult or impossible to study in vivo. Second, samples can be imaged with minimal handling and without invasive procedures, enabling study of rare specimens that would not be appropriate to dissect. Third, MRI investigations can be performed in whole brain samples, rather than excised tissue sections. This makes post-mortem MRI ideally placed to characterise macroscopic brain structure, long-range structural connectivity and tissue microstructure in species that are not traditional experimental models, and in particular rare species where very few brain samples may be available (Berns \& Ashwell, 2017; Bhagwandin et al., 2017; Grewal et al., 2020; Mars et al., 2014).

MRI data from multiple species allows one to formally compare brain organization, important for large-scale comparative neuroscience which has traditionally relied on very limited measures (e.g. whole or regional brain size measures of brain organization) (Mars et al., 2014). The ability to acquire data from whole brains opens up 
bioRxiv preprint doi: https://doi.org/10.1101/2021.06.21.449154; this version posted September 1, 2021. The copyright holder for this

preprint (which was not certified by peer review) is the author/funder, who has granted bioRxiv a license to display the preprint in perpetuity. It is made available under aCC-BY 4.0 International license.

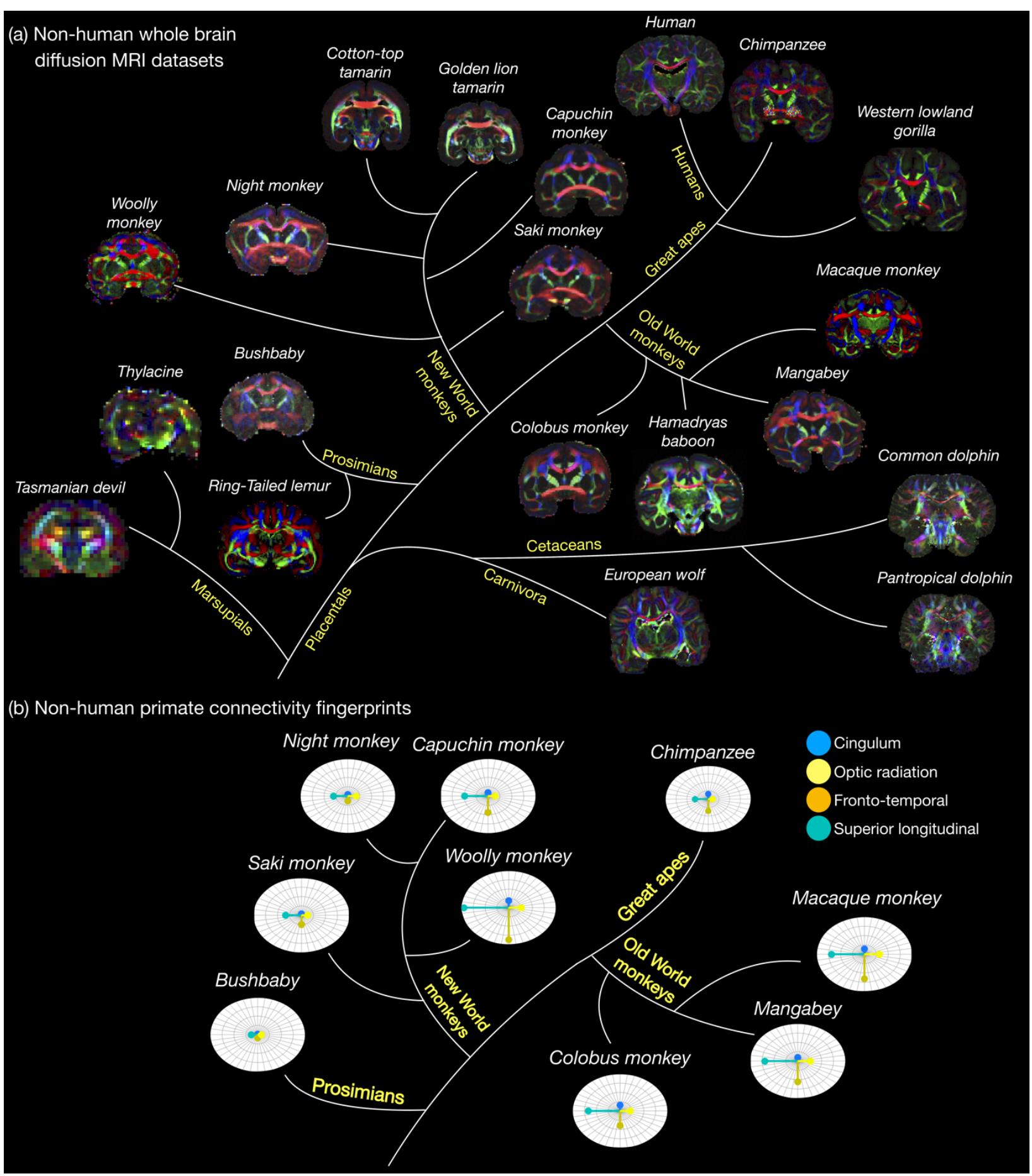

Figure 2: The Digital Brain Zoo. The first release of the Digital Brain Zoo provides whole-brain MRI datasets spanning multiple species and taxonomic ranks. Notably, we provide whole-brain diffusion MRI datasets from fourteen non-human primate species, with samples selected for their high quality and to ensure sampling of all major branches of the primate evolutionary tree (Prosimian, New World monkey, Old World monkey, Great Ape). (b) compares the relative volume of four tracts derived from nine non-human primate post-mortem datasets provided in the Digital Brain Zoo (Bryant et al., 2021), where increased distance from the centre corresponds to an increased volume.

the possibility of elucidating principles of neural diversity across larger orders of mammalian species (Friedrich et al., 2021), and create between-species mappings to formally identify homologies and quantify unique aspects of any given brain (Mars et al., 2018). This also allows one to improve translational neuroscience by better understanding the relationship between the human brain and that of model species (e.g. macaque, marmoset, rat, mouse). 
The Digital Brain Zoo provides access to post-mortem imaging datasets in non-human species covering multiple taxonomic ranks (Fig. 2a), including non-human primate species (Bryant et al., 2021; Roumazeilles et al., 2020, 2021), Carnivora (Grewal et al., 2020), Marsupials (Berns \& Ashwell, 2017) and Cetaceans (Berns et al., 2015). As with other collections in the Digital Brain Bank, the Digital Brain Zoo currently focuses primarily on whole brain diffusion MRI. These datasets offer multiple pathways of investigation in comparative neuroanatomy, for example, through the examination of structural connections across brains (Fig. 2b) (Bryant et al., 2021). Furthermore, our developments in imaging large post-mortem samples have enabled us to acquire a number of high-quality post-mortem imaging datasets in species with brains that are too large to fit into specialised pre-clinical MRI systems, conventionally used to improve image quality in post-mortem MRI (see Discussion). Future releases to the Digital Brain Zoo will continue to build upon our strong links with postmortem brain banks and collaborators to expand the species range. More primate species are currently in preparation, as are extensions into orders Carnivora and Rodentia.

\section{Digital Pathologist}

Datasets within the Digital Pathologist provide a new direction for examining neuropathology and MRIpathology correlates.

One of the biggest challenges in the use of MRI clinically is the lack of specificity to disease mechanisms. Many neurological diseases are characterised by changes at the cellular and subcellular level, which cannot be directly visualised with the limited resolution of MRI. Nevertheless, MRI contrast can be made sensitive to cellular-level phenomena that are relevant to disease. Acquisition of MRI and histology in the same tissue enables us to relate microscopic changes in the neural microenvironment to MRI image contrast. The primary aim of the Digital Pathologist is to facilitate these cross-scale comparisons, imaging brain tissue associated with a neurological disease.

Such data are provided in the Human ALS MRI-Histology dataset (Fig. 3a), which aims to identify how neuropathological changes in amyotrophic lateral sclerosis (ALS) give rise to altered MRI contrast, and answer specific questions related to ALS pathology. The Human ALS MRI-Histology dataset provides whole-brain multimodal MRI and selective histology in a cohort of 12 ALS (diagnosis during lifetime, confirmed ALS neuropathology) and 3 control (no known neuropathology) brains (Pallebage-Gamarallage et al., 2018) provided by the Oxford Brain Bank. MRI data includes diffusion, structural, quantitative susceptibility maps (via quantitative susceptibility mapping, QSM), and quantitative T1, T2 and T2* maps. Histology includes markers for proteinopathy (pTDP-43), microglia (CD68 and IBA1), myelin (PLP), neurofilaments (SMI-312) and iron (ferritin) in order to detect changes in a range of microstructures within cortical and subcortical regions (anterior cingulate cortex, corpus callosum, hippocampus, primary motor cortex and visual cortex) associated with different proposed stages of ALS disease progression (Jucker \& Walker, 2013).

Different MRI modalities have known sensitivities to different components of the cellular environment. Combined with multi-modal histology, these data provide the opportunity to relate neuropathologicallyinduced changes in tissue microstructure to MRI image contrast. While these aims could be partially achieved by dissecting and scanning sub-regions of the brain, our approach of scanning whole post-mortem brains enables us to investigate neuropathological spread across the entire brain (Jucker \& Walker, 2013). This facilitates investigations across long-range fibre-tracts associated with pathology (Fig. $3 \mathrm{~b}$ ), or microstructural changes in multiple brain regions (Fig. 3c). Notably, these analyses are being facilitated by accurate crossmodality image coregistrations (Huszar et al., 2019), enabling us to perform pixelwise evaluations and integrate structural analyses to identify how pathology influences MR image contrast (Fig. $3 \mathrm{~d}$ ) in a subset of brain regions associated with different proposed stages of ALS disease progression (Jucker \& Walker, 2013). MRI data for the Human ALS MRI-Histology dataset for all 12 ALS and 3 control brains is immediately available to download, alongside a subset of histology data. Remaining histology data and MRI-histology coregistrations are being actively curated for future release to the Digital Brain Bank.

Tview

The Digital Brain Bank website enables users to browse a subset of data easily. A key feature of many datasets is that they contain both MRI and microscopy data. Few available viewers, whether downloadable or online, support both MRI and microscopy file formats, creating a barrier of entry for potential users. Moreover, 2D 
bioRxiv preprint doi: https://doi.org/10.1101/2021.06.21.449154; this version posted September 1, 2021. The copyright holder for this preprint (which was not certified by peer review) is the author/funder, who has granted bioRxiv a license to display the preprint in perpetuity. It is made available under aCC-BY 4.0 International license.

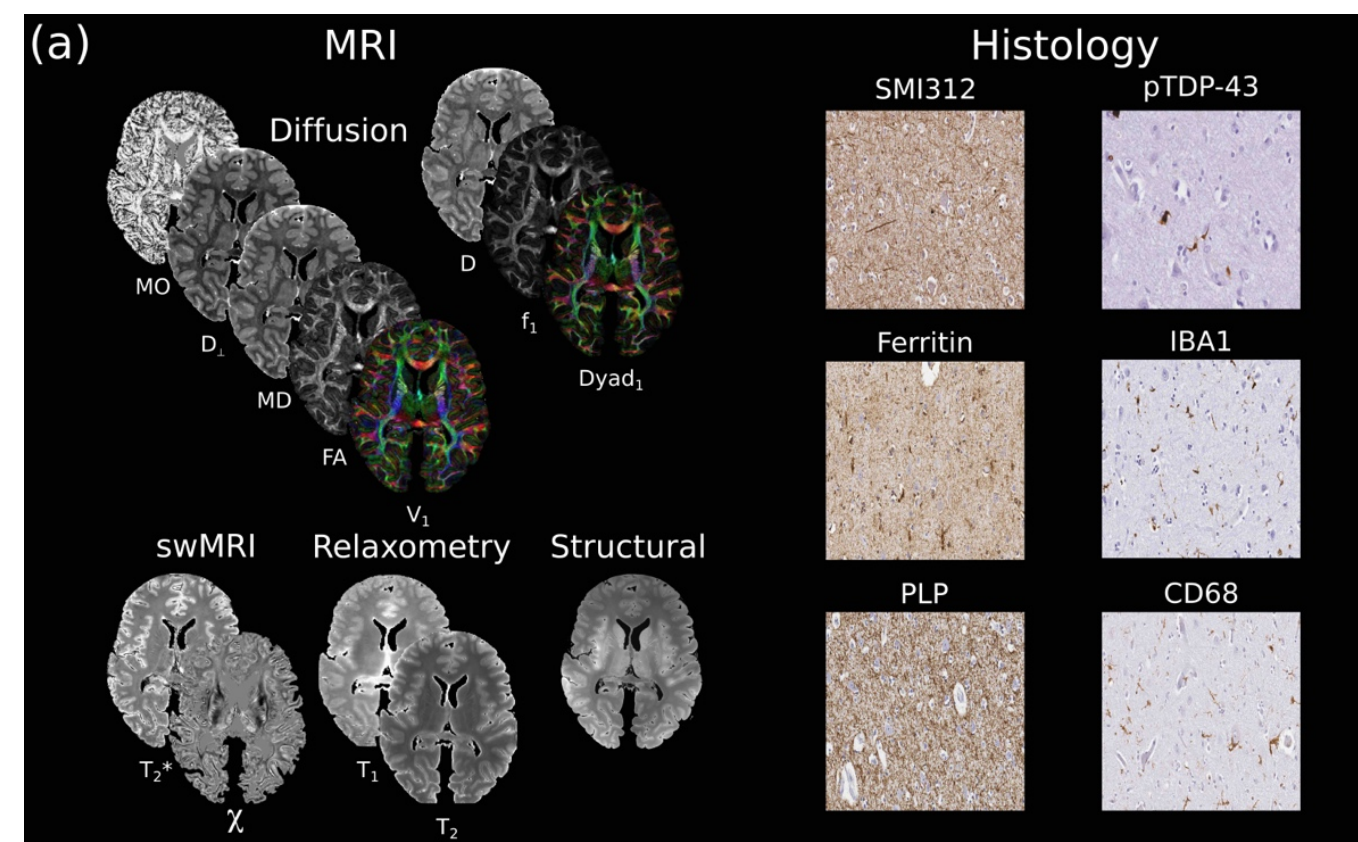

(b) Tractography - ALS staging regions

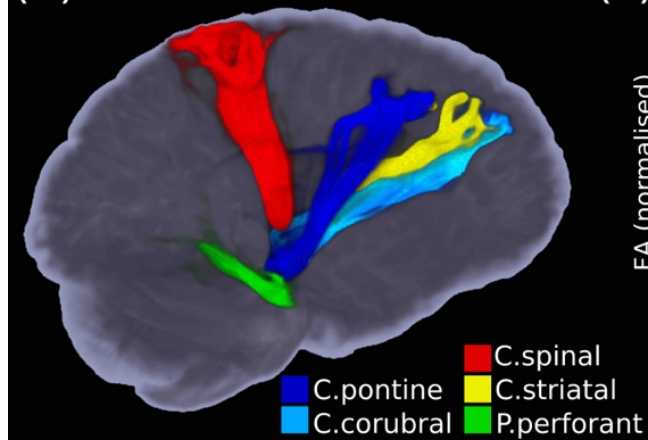

(C) FA across the corpus callosum (ALS vs controls)

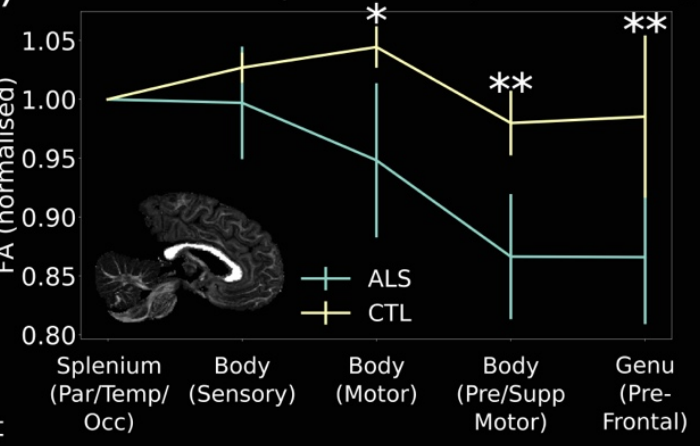

(d) MRI-Histology coregistration (visual cortex)

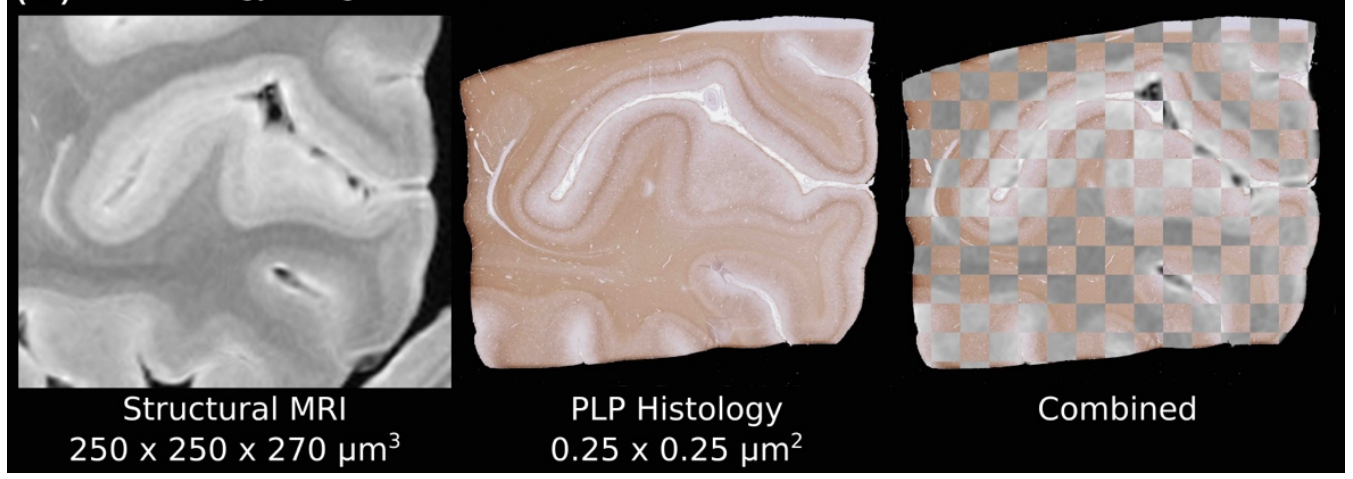

Figure 3: The Digital Pathologist. One of the key aims of the Digital Pathologist is the examination of neuropathological spread in neurological disease. The Human ALS MRI-Histology dataset (a) facilitates these investigations, combining wholebrain multi-modal MRI and histology (selected brain regions) in a cohort of 12 ALS and 3 control brains. (b) displays the reconstruction of five white matter pathways associated with different ALS stages in a single post-mortem brain (Kassubek et al., 2014). Comparisons between ALS and control brains over the corpus callosum of the cohort (c) reveals changes in fractional anisotropy (FA, normalised to Par/Temp/Occ lobe), with biggest changes associated with motor and pre-frontal regions (Hofer \& Frahm, 2006) $(*=p<0.05 ; * *=p<0.05$ following multiple comparison correction) (full details of the corpus callosum analysis provided in Supporting Information). Accurate MRI-histology coregistrations facilitates crossmodality comparisons, and (d) displays an example MRI-histology coregistration over the visual cortex of a single ALS brain achieved using the Tensor Image Registration Library (TIRL) (Huszar et al., 2019). $V_{1}=$ Principal Diffusion Direction, FA = Fractional Anisotropy, $\mathrm{MD}=$ Mean Diffusivity, $\mathrm{D}_{\perp}=$ radial diffusivity, $\mathrm{MO}=$ Mode from diffusion tensor output, $\mathrm{Dyad}_{1}=$ principal dyad orientation, $\mathrm{f}_{1}=$ principal fibre fraction and $\mathrm{D}=$ diffusivity from ball $\& 2$-sticks output, swMRI = susceptibilityweighted MRI, $\chi=$ magnetic susceptibility. Details of stain contrasts in (b) and (d) provided in Table 1. 
microscopy datasets are extremely high resolution: single images can exceed $10,000,000,000$ pixels, running to gigabytes in size.

We aim to provide online viewing of microscopy and MRI data using standard internet browsers, with a viewer that can handle data at very different spatial scales, provide flexible image overlays, and support colour visualisation for diffusion-derived measures, PLI and multiple histological counterstains. Unable to identify existing software with these features we developed a web-based image viewer, Tview. Tview is based on software originally used to display satellite imagery at multiple elevations, and enables real-time visualisation, interaction (zooming/panning), and flexible overlays of different modalities in a single 2D plane of MRI and microscopy data. Visualisation of multimodal (i.e. MRI and microscopy) datasets on the Digital Brain Bank website is achieved with Tview. An example Tview implementation is available at open.win.ox.ac.uk/DigitalBrainBank/\#/tileviewer, where cross-modality coregistrations were performed using the Tensor Image Registration Library (TIRL) (Huszar et al., 2019) and FNIRT (Andersson et al., 2007; Jenkinson et al., 2012), both available as part of FSL. Code for Tview, the website and server implementation are available at https://git.fmrib.ox.ac.uk/thanayik/dbb.

\section{Requirements for Data Access and Referencing Datasets}

The Digital Brain Bank has been designed to minimise the burden on the user to download datasets, within ethical constraints. Some are available to directly download on the Digital Brain Bank website, such as the Human Callosum MRI-PLI-Histology dataset (Digital Anatomist) provided in the first data release. For many datasets, we have developed conditions of use terms via a material transfer agreement (MTA), which users agree to prior to access. The MTAs are primarily designed to ensure that datasets are used for research/educational purposes, to prevent misuse, and to satisfy funding requirements.

For datasets restricted by MTAs, when possible a subset of example data (e.g. data from a single subject) is available to download directly on the website. Upon signing the MTA, users will be granted access to the full dataset. MTAs are currently approved by the University of Oxford. This is currently achieved via the email address provided with each dataset on the Digital Brain Bank website. We are actively exploring alternatives to streamline this process.

Upon downloading Digital Brain Bank datasets, users agree to acknowledge the source of the data in any outputs. Users are asked to cite the original study for any given dataset and the Digital Brain Bank. Details of associated publications and citation instructions are available on an information page associated with each dataset on the Digital Brain Bank website.

\section{Discussion}

The Digital Brain Bank has been specifically designed to cover the breadth of spatial scales and modalities encountered in post-mortem imaging. Features on the website facilitate data discovery, with users able to interact with a subset of available datasets prior to access. The Digital Brain Bank is envisioned as a growing resource reflecting a range of post-mortem neuroimaging projects. Alongside the first release, we aim to first bring together a large number of datasets that have been accumulated over the past decade at the University of Oxford. Beyond this, the Digital Brain Bank will be the primary resource to release new post-mortem imaging datasets associated with both departmental and collaborative projects. We envisage the Digital Brain Bank as providing complementary datasets to existing open-science initiatives in human and non-human neuroimaging (for example, PRIME-DE (Milham et al., 2018)).

\section{Post-mortem MRI}

Post-mortem MRI facilitates the non-invasive investigation of brain anatomy, tissue composition and structural connectivity through the acquisition of high-resolution datasets and subsequent microscopy comparisons. Despite this potential, post-mortem MRI remains a relatively niche approach, in part due to technical challenges and need for multi-disciplinary expertise. In order to provide post-mortem MRI as an experimental technique to neuroscientists in Oxford, we have had to develop a broad range of underpinning technologies, including: (i) pulse sequences that provide high quality data under the harsh imaging conditions of post- 
bioRxiv preprint doi: https://doi.org/10.1101/2021.06.21.449154; this version posted September $1,2021$. The copyright holder for this preprint (which was not certified by peer review) is the author/funder, who has granted bioRxiv a license to display the preprint in perpetuity. It is made available under aCC-BY 4.0 International license.

\begin{tabular}{|c|c|c|c|}
\hline Category & Dataset(s) & $\begin{array}{l}\text { Acquisition } \\
\text { location }\end{array}$ & MRI scanner \\
\hline $\begin{array}{l}\text { Digital } \\
\text { Anatomist }\end{array}$ & $\begin{array}{l}\text { Human High-Resolution } \\
\text { Diffusion MRI-PLI }\end{array}$ & $\begin{array}{l}\text { University } \\
\text { of Oxford }\end{array}$ & $\begin{array}{c}\text { Siemens 7T Magnetom } \\
\text { 32-channel receive/1-channel transmit head coil (Nova } \\
\text { Medical) }\end{array}$ \\
\hline $\begin{array}{l}\text { Digital } \\
\text { Anatomist }\end{array}$ & $\begin{array}{l}\text { Human Callosum MRI- } \\
\text { PLI-Histology }\end{array}$ & $\begin{array}{l}\text { University } \\
\text { of Oxford }\end{array}$ & $\begin{array}{l}\text { 9.4T } 160 \mathrm{~mm} \text { horizontal bore VNMRS preclinical MRI system } \\
100 \mathrm{~mm} \text { bore gradient insert (Varian Inc) } \\
26 \mathrm{~mm} \text { ID quadrature birdcage coil (Rapid Biomedical GmbH) }\end{array}$ \\
\hline $\begin{array}{l}\text { Digital Brain } \\
\text { Zoo }\end{array}$ & Non-Human Primates & $\begin{array}{l}\text { University } \\
\text { of Oxford }\end{array}$ & $\begin{array}{c}\frac{\text { Baboon, Chimpanzee, Gorilla }}{\text { Siemens 7T Magnetom }} \\
\text { 28-channel receive/1 channel transmit knee coil (QED) } \\
\text { 7T magnet with Agilent Direct-Drive console } \\
\text { 72mm ID quadrature birdcage RF coil (Rapid Biomedical } \\
\text { GmbH) }\end{array}$ \\
\hline $\begin{array}{l}\text { Digital Brain } \\
\quad \text { Zoo }\end{array}$ & Marsupials & $\begin{array}{l}\text { Emory } \\
\text { University }\end{array}$ & $\begin{array}{l}\text { Siemens 3T Trio } \\
\text { 32-channel receive/1-channel transmit head coil }\end{array}$ \\
\hline $\begin{array}{l}\text { Digital Brain } \\
\text { Zoo }\end{array}$ & Cetaceans & $\begin{array}{l}\text { Emory } \\
\text { University }\end{array}$ & $\begin{array}{c}\frac{2 \times \text { Tasmanian devil and } 1 \times \text { Thylacine }}{\text { Siemens } 3 T \text { Trio }} \\
\text { 32-channel receive/1-channel transmit head coil } \\
\frac{1 \times \text { Thylacine }}{\text { Bruker 9.4 T BioSpec preclinical MR system }}\end{array}$ \\
\hline $\begin{array}{l}\text { Digital Brain } \\
\text { Zoo }\end{array}$ & Carnivora & $\begin{array}{l}\text { University } \\
\text { of Oxford }\end{array}$ & $\begin{array}{l}\text { Siemens 7T Magnetom } \\
\text { 28-channel receive/1 channel transmit knee coil (QED) }\end{array}$ \\
\hline $\begin{array}{c}\text { Digital } \\
\text { Pathologist }\end{array}$ & $\begin{array}{l}\text { Human ALS MRI- } \\
\text { Histology }\end{array}$ & $\begin{array}{l}\text { University } \\
\text { of Oxford }\end{array}$ & $\begin{array}{c}\text { Siemens 7T Magnetom } \\
\text { 32-channel receive/1-channel transmit head coil (Nova } \\
\text { Medical) }\end{array}$ \\
\hline
\end{tabular}

Table 2: Acquisition site and MRI scanner associated with all projects in the first release to the Digital Brain Bank.

mortem tissue (McNab et al., 2009; Miller et al., 2011); (ii) analyses that account for the signal formation mechanisms of these sequences (Tendler, Foxley, Cottaar, et al., 2020) or properties unique to post-mortem tissue (Tendler, Qi, et al., 2020); (iii) experimental approaches that enable the use of ultra-high field MRI to increase SNR for high-resolution imaging (Foxley et al., 2014; Tendler, Foxley, Hernandez-Fernandez, et al., 2020); (iv) development of custom sample holders to maximise SNR and minimise imaging artefacts (Supporting Information Figs. S1 and S2); (v) tools for aligning small 2D microscopy images into 3D whole-brain MRI (Huszar et al., 2019); (vi) strategies for co-analysing MRI and microscopy data (A. F. Howard et al., 2019; Mollink et al., 2017) and (vii) techniques for between-species comparisons (Eichert et al., 2020; Mars et al., 2018).

The investment of multidisciplinary expertise and effort required to create these datasets will inevitably be a barrier to similar studies elsewhere. The Digital Brain Bank makes our data openly available to researchers worldwide to enable a much broader range of investigations. Considerable work has been performed to process images in a manner which users can immediately incorporate into their own analyses (e.g. diffusion tensor and ball \& sticks signal models) reducing user burden to develop their own data processing methods. Further details of these outputs are provided in the Methods section.

Here, we provide an overview of how we overcame challenges associated with imaging these samples, notably those associated with imaging large post-mortem brains. Datasets were acquired over many years from multiple imaging sites, resulting in evolving experimental setup, acquisition and processing methods between datasets. To avoid an exhaustive list of different imaging approaches, below we describe the methodology undertaken for acquisitions performed at the University of Oxford, where the majority of datasets in the first release were acquired. Details of the acquisition location and scanner used for all datasets are provided in Table 2. 


\section{Choice of MRI scanner}

Specialised RF coils and imaging gradients facilitate the acquisition of high-resolution, high-SNR post-mortem MRI datasets. Pre-clinical systems often deliver in this space, notably with powerful gradient sets, and where possible should be adopted for post-mortem imaging. Specifically, post-mortem tissue that has undergone chemical preservation with aldehyde solutions (e.g. formalin) is characterised by short relaxation time constants (T1, T2 and T2*) (Birkl et al., 2016, 2018; Dawe et al., 2009; Kamman et al., 1985; Nagara et al., 1987; Pfefferbaum et al., 2004; Shepherd et al., 2009; Thelwall et al., 2006), and low diffusivity (H. E. D'Arceuil et al., 2007; Shepherd et al., 2009; Sun et al., 2003, 2005; Thelwall et al., 2006) when compared to in vivo tissue. Powerful gradient sets provide rapid signal sampling and strong diffusion weighing, which boosts SNR versus conventional gradients (Dyrby et al., 2011; Roebroeck et al., 2019) in this environment.

Broadly speaking, post-mortem MRI data provided in the first release to the Digital Brain Bank can be categorised into two experimental designs. Small post-mortem samples (e.g. small NHP brains and excised tissue blocks) were scanned using specialised pre-clinical systems with powerful gradient sets. At the University of Oxford, these scans were performed with either a 7T pre-clinical system with Agilent DirectDrive console (Agilent Technologies, CA, USA), or a 9.4 T $160 \mathrm{~mm}$ horizontal bore VNMRS preclinical MRI system equipped with a $100 \mathrm{~mm}$ bore gradient insert (Varian Inc, CA, USA).

Whole brains of larger species do not physically fit into these pre-clinical systems (maximum sample diameter 7-8 cm) and can only be accommodated in human scanners. These systems often have comparatively low gradient strengths, reducing the available SNR. At the University of Oxford, these brains were scanned on a Siemens 7T Magnetom human scanner. Here, we addressed the imaging environment of fixed, post-mortem tissue and comparatively low gradient-strengths by investing in alternative MR sequences to increase SNR. Further details of this are provided below.

\section{Sample preparation}

All brains and tissue samples in the first release of the Digital Brain Bank were chemically fixed using aldehyde solutions (e.g. formalin) to prevent decomposition (H. D’Arceuil \& de Crespigny, 2007) and minimise deformation during the course of scanning. All fixed non-human brains and excised tissue blocks scanned at the University of Oxford were prepared by soaking the samples in phosphate buffered saline (PBS) prior to scanning, which increases image SNR by raising $T_{2}$-values closer to those found in vivo (Shepherd et al., 2009). This was not performed in whole human brains, as brain size necessitates a soaking time of multiple weeks for the buffer fluid to penetrate throughout tissue (Dawe et al., 2009; Tendler, Qi, et al., 2020; Yong-Hing et al., 2005) which was incompatible with our experimental design. We note that soaking tissue for an insufficient time can lead to artificial 'boundaries' in resulting images, where PBS has not penetrated into deep tissue (Miller et al., 2011).

\section{Scanning medium and sample holder}

Susceptibility artefacts (arising due to air-tissue or air-medium interfaces) can be exacerbated in post-mortem imaging without an appropriate scanning medium. All samples scanned at the University of Oxford were imaged in a proton-free susceptibility-matched fluid (Fomblin LC08, Solvay Solexis; or Fluorinert FC-3283, 3M). The choice of a proton-free fluid means that there is no signal outside of the brain, bringing the additional advantage of minimising the required field-of-view for any acquisitions, and addressing scaling issues arising from a bright background signal.

For whole human brain imaging, we built a two-stage custom holder (Supporting Information Fig. S1), which has become the standard for all of our whole-brain human imaging experiments. The holder was designed to fit into a 32-channel receive/1-channel transmit head coil (Nova Medical), securing brains throughout the acquisition, and contains a spherical cavity to minimise field-inhomogeneities across the brain. The holder enables brains to be placed in a consistent position (equivalent to an in vivo supine scan), minimising variability of $\mathrm{B}_{0}$-orientation dependent effects (e.g. susceptibility anisotropy (Liu, 2010)), as well as avoiding any potential motion. While motion is clearly less problematic than in vivo, samples must be well-secured, as even small motions can give rise to coregistration challenges and artefacts across the acquisition period (often 
$>24$ hours). All human brains were scanned in this holder, with the exception of the Human High-Resolution Diffusion MRI-PLI dataset (data acquired prior to holder construction). Full information on this experimental set up is provided in (Wang et al., 2020).

Large non-human brains scanned at the University of Oxford (Gorilla, Chimpanzee, Wolf, Baboon) were placed inside a 28 channel receive/ 1 channel transmit knee coil (QED) to boost SNR (smaller distance between sample and the imaging coil). These brains were placed inside a cylindrical brain holder (Supporting Information Fig. S2), with a cylindrical cavity that is compatible with the shape of the knee coil, and is a shape that minimises $B_{0}$ field inhomogeneities. Small non-human brains/excised tissue blocks scanned on pre-clinical systems were placed in simpler containers, e.g. syringes filled with fluorinert.

\section{Structural MRI}

Structural MRI enables the delineation of fine tissue structures and cortical surface reconstruction through high contrast, high-resolution imaging datasets. However, convergence of T1 relaxation times for grey and white matter in formalin fixed post-mortem tissue leads poor contrast with conventional T1-weighted structural protocols (Miller et al., 2011). All structural MRI datasets available in the first data release were acquired using a balanced SSFP (bSSFP) sequence, which has demonstrated excellent post-mortem grey/white matter contrast. bSSFP signal forming mechanisms lead to an extremely high SNR-efficiency (even when considering the reduced $\mathrm{T} 1$ and $\mathrm{T} 2$ of post-mortem tissue), affording the acquisition of ultra-high resolution (< $500 \mu \mathrm{m}$ ) imaging volumes to delineate fine tissue structures.

\section{Diffusion MRI}

Post-mortem diffusion MRI is particularly challenging due to the MR-relevant properties of fixed tissue, with reductions in measured relaxation time constants T1, T2 and T2* (Birkl et al., 2016, 2018; Dawe et al., 2009; Kamman et al., 1985; Nagara et al., 1987; Pfefferbaum et al., 2004; Shepherd et al., 2009; Thelwall et al., 2006), and diffusivity (H. E. D'Arceuil et al., 2007; Shepherd et al., 2009; Sun et al., 2003, 2005; Thelwall et al., 2006) routinely reported in literature.

To achieve high SNR in these conditions, specialised pre-clinical systems and tissue preparation methods are often required (Roebroeck et al., 2019), with many groups focusing on tissue sections that can be scanned on rodent scanners with specialised hardware (Beaujoin et al., 2018; Calabrese et al., 2015). Unfortunately, size constraints restrict the use of pre-clinical systems to small post-mortem tissue samples (e.g. small NHP brains or excised tissue blocks). As described above, large whole brains do not physically fit into pre-clinical systems, and can only be accommodated in human scanners. These systems often have comparatively low gradient strengths; combined with conventional methods (e.g. diffusion-weighted spin echo, DW-SE), this can lead to low-SNR diffusion imaging volumes.

Over the past decade, our lab has invested considerably into the use of an alternative diffusion imaging technique, diffusion-weighted steady-state free precession (DW-SSFP) (Kaiser, Bartholdi, \& Ernst, 1974; Le Bihan, 1988; K. D Merboldt et al., 1989; K. D. Merboldt, Hxnicke, Gyngell, Frahm, \& Bruhn, 1989), to achieve high-SNR datasets in large post-mortem samples. DW-SSFP is well suited to the environment of fixed postmortem tissue, achieving strong diffusion weighting and rapid signal sampling, even when hardware achieves limited gradient amplitudes and when $\mathrm{T}_{2}$ values are low (McNab et al., 2009; Vasung et al., 2019; Wilkinson et al., 2016). The DW-SSFP sequence has demonstrated improved SNR-efficiency compared to conventional DWSE when imaging post-mortem tissue (Miller et al., 2012), further enhanced at ultra-high field (7T) (Foxley et al., 2014). For more details regarding DW-SSFP, please see (McNab \& Miller, 2010).

Broadly, two separate diffusion imaging approaches were used for the first release of data to the Digital Brain Bank. Small brains and excised tissue blocks imaged on pre-clinical systems were scanned using conventional DW-SE sequences, where tissue preparation and powerful diffusion gradients provide imaging volumes with high SNR. Diffusion imaging for larger post-mortem samples scanned on a human scanner (Siemens 7T Magnetom) was performed using DW-SSFP.

To facilitate cross-dataset comparisons, the majority of diffusion datasets from the Digital Brain Bank provide derived diffusivity estimates in the form of diffusion tensor and/or ball \& sticks model parameters (Behrens et 
al., 2007). Whilst there are a number of standard software packages available for DW-SE data, this was achieved for DW-SSFP datasets using a custom imaging pipelines incorporating the full DW-SSFP model (including $\mathrm{T}_{1}, \mathrm{~T}_{2}$ and $\mathrm{B}_{1}$ dependencies) (Tendler, Foxley, Hernandez-Fernandez, et al., 2020).

There are some differences between derived diffusivity estimates from DW-SSFP and DW-SE data. Importantly, the DW-SSFP signal does not have a well-defined b-value (McNab \& Miller, 2010; Tendler, Foxley, Cottaar, et al., 2020). For all DW-SSFP datasets acquired in whole human brains (e.g. the Human ALS MRI-Histology dataset, the Human High-Resolution Diffusion MRI-PLI dataset), we utilized a recently proposed approach to transform DW-SSFP datasets acquired at two flip angles into equivalent measurements at a single, welldefined b-value (Tendler, Foxley, Cottaar, et al., 2020; Tendler, Foxley, Hernandez-Fernandez, et al., 2020). This facilitates within dataset comparisons, alongside comparisons with datasets acquired with the DW-SE sequence. However, this approach was not possible for the non-human DW-SSFP datasets due to differences in the acquisition protocol. Although the diffusivity estimates for the non-human DW-SSFP datasets directly relate to the underlying diffusivity of tissue, the DW-SSFP signal forming mechanisms lead to varying effective b-values within and between these datasets (Tendler, Foxley, Cottaar, et al., 2020). Conservatively, we recommend the non-human DW-SSFP datasets to be primarily used for structural connectivity (e.g. tractography) investigations.

More generally, differences in the number of diffusion directions, choice of b-value (for DW-SE and DW-SSFP transformed datasets), imaging resolution, and SNR exist across datasets, a result of available scanning hardware, scanning time, experimental design and sample properties (e.g. type of fixative used and size of the brain). These limitations can lead to differences in resulting diffusivity estimates and should be considered when performing comparisons across different datasets in the Digital Brain Bank. Full details of the acquisitions are provided in the original publications, alongside information on the Digital Brain Bank website and dataset downloads.

\section{Other sequences}

Quantitative T1 and T2 maps are provided with the post-mortem whole-brain human datasets, acquired using conventional turbo inversion-recovery (TIR) and turbo spin-echo (TSE) sequences. Notably, T1-convergence of grey and white matter in fixed post-mortem tissue leads to low contrast on T1 maps, as described in the Structural MRI section above. T1 maps were fitted assuming mono-exponential signal recovery. T2 maps were processed using an extended phase graph (EPG) fitting scheme, which accounts for B1-inhomogeneity at 7T (details of acquisition and processing are described in (Tendler, Qi, et al., 2020)).

Whole human brain quantitative $\mathrm{T} 2 *$ and quantitative susceptibility maps are available in a subset of brains provided with the Human ALS MRI-Histology dataset. These data were acquired using a multi-echo gradientecho sequence and processed following the procedure in (Wang et al., 2020).

\section{Cross-scale comparisons}

Post-mortem imaging experiments combining MRI and microscopy are routinely used to validate the origins of image contrast. However, these comparisons are often restricted to simple summary statistics (e.g. ROI averages), rather than utilising all the available data through pixelwise comparisons and structural analyses (Mollink et al., 2017). These more detailed approaches are facilitated by accurate cross-modality coregistrations, a considerable challenge given differences in image contrast and tissue deformations arising from microscopy processing (Huszar et al., 2019; Iglesias et al., 2018; Ohnishi et al., 2016). These challenges are further exacerbated when considering small tissue sections excised from large post-mortem samples, where the corresponding microscopy sampling region must be identified in a 3D imaging volume.

To address this, our group has developed the Tensor Image Registration Library (TIRL), a novel MR-microscopy coregistration toolbox achieving automated cross-modality imaging coregistrations (Huszar et al., 2019). Notably, this approach can be automated for coregistering 3D MR volumes to 2D microscopy images, typically given a set of sequential block-face photographs taken during the tissue dissection process. These coregistrations are being performed for the Human ALS MRI-Histology (Digital Pathologist) dataset (Fig. 3d), and are currently available for two brains. Remaining coregistrations are being actively curated, and will be provided in a future release to the Digital Brain Bank. 


\section{Methods}

\section{Web Development and Tview}

The Digital Brain Bank is a web application made up of individual service components, created using a combination of open source software. Services include the dataset downloader, the website and Tview. The web application is hosted on our own server hardware, and the various services of the application are orchestrated using container management system Docker (docker.com).

The core website and user interface were created using Vue (vuejs.org), a frontend web framework for composing reusable application components. The Vue website communicates with multiple backend services (via HTTP requests) to retrieve information (e.g. which Tview tiles to display, which datasets are available for download). The reactive web application ensures that changes to website are seen in real time, or on the next possible page reload.

Tview provides real-time zooming/panning of high-resolution microscopy and MRI overlays using leafletjs, a software library originally used to display satellite imagery at multiple elevations. We have adapted our microscopy datasets to be compatible with this software library in order to take advantage of the features it offers. This is achieved by converting images into tiles at multiple zoom levels (via libvips), and uploading these tiles to the local web server. Only the relevant subset of tiles are downloaded when users interact with images using Tview. Individual tiles comprise a small file size, facilitating real-time interaction.

Continued use of the Digital Brain Bank requires a simple process for collaborators to upload post-mortem datasets, and an application administration layer is under active development to facilitate this process. Code for the Digital Brain Bank web application is available at https://git.fmrib.ox.ac.uk/thanayik/dbb.

\section{Datasets}

The Digital Brain Bank is not designed as a stand-alone resource - when possible, datasets are associated with available publications which extensively describe the methodology used. This approach facilitates the referencing of available datasets, and similarly ensures that sufficient detail is provided on how data were acquired and processed. A list of the associated publications with the first release of datasets is provided in Table 1. However, as part of the first release of the Digital Brain Bank, we provide a human dataset which has not yet been described in literature, the Human High-Resolution Diffusion MRI-PLI dataset. We additionally provide four new species datasets in for the Digital Brain Zoo, the Hamadryas baboon, Golden Lion Tamarin, Cotton-Top tamarin and European wolf. A full description of the acquisition and data processing for these data are provided in the Supporting Information.

\section{Conditions for Data Uploading}

All datasets hosted on the Digital Brain Bank are associated with projects performed at the University of Oxford, or in collaboration with members of the University of Oxford. Datasets considered a continuation of research after a formal collaboration with the University of Oxford will also be considered for upload. Information regarding the primary contributors to the dataset are explicitly stated on the Digital Brain Bank website. All projects must have been granted ethical approval from the relevant brain banks and departmental ethics boards. Datasets will be shared on the Digital Brain Bank website on the condition that data providers do not require co-authorship for any subsequent outputs based on the use of the datasets alone.

\section{Types of Data Provided}

There are no strict criteria regarding the types of post-mortem data released to the Digital Brain Bank, nor the structure of the dataset. This direction has been chosen to account for the variety of independent projects that will be uploaded. However, the Digital Brain Bank aims to facilitate the investigation of research hypotheses designed by the user, rather than replicating previously published studies. To reflect this, we primarily provide 
curated datasets to facilitate these analyses, as opposed to outputs associated with the results of specific projects (e.g. tractography-derived maps).

First and foremost, the Digital Brain Bank is a data sharing resource. Details of the acquisition and processing methodology associated with each dataset is provided with the accompanying manuscripts, on the Digital Brain Bank website (Information page associated with each project), and with the downloaded dataset. However, data hosts are encouraged to provide pre-processing code when available. This code will be linked with each dataset on the Digital Brain Bank website (on the Information page), or packaged with the dataset download.

In the first release to the Digital Brain Bank, curated MRI outputs comprise of diffusion model estimates (e.g. diffusion tensor and ball \& sticks) (Behrens et al., 2007), averaged structural MRI datasets, quantitative relaxometry (e.g. quantitative $\mathrm{T} 1, \mathrm{~T} 2$ and $\mathrm{T} 2 *$ maps), and quantitative susceptibility maps. Curated unprocessed data is additionally provided when there are clear routes of investigation (e.g. diffusion MRI volumes to investigate alternative diffusion models).

These curated outputs reduce the burden on the user to develop their own processing pipelines, of particular importance when considering datasets acquired with alternative sequences not addressed with commonly used imaging software (e.g. DW-SSFP) (Tendler, Foxley, Hernandez-Fernandez, et al., 2020), or datasets which required specialised fitting approaches (e.g. extended phase graph fitting required for T2 mapping at 7T, used in the Human ALS MRI-Histology and Human High-Resolution Diffusion MRI-PLI datasets) (Tendler, Qi, et al., 2020; Weigel, 2015).

For multi-modal (MRI and microscopy) datasets in the first release (Human High-Resolution Diffusion MRI-PLI, Human Callosum MRI-PLI-Histology and Human ALS MRI-Histology dataset), original high-resolution microscopy images are provided. A full set of coregistered data to enable MRI-microscopy voxelwise comparisons via TIRL (Huszar et al., 2019) are being actively curated for future release (Fig. 3d). These are currently available for two brains of the Human ALS MRI-Histology dataset.

Metadata specific to the analysis of post-mortem tissue (e.g. fixative type, post-mortem interval etc), or relevant to distinguishing individual datasets in a cohort study (e.g. control brain, or brain with a neurological disease) is provided when available.

\section{Ethics Statement}

All human post-mortem datasets described in the first release to the Digital Brain Bank used tissue provided by the Oxford Brain Bank, a research ethics committee (REC) approved, HTA regulated research tissue bank. The studies were conducted under the Oxford Brain Bank's generic Research Ethics Committee approval (15/SC/0639).

There are four new species datasets (Hamadryas baboon, Golden Lion tamarin, Cotton-Top tamarin, and European wolf) provided in the first release to the Digital Brain Bank which have not been previously described in literature. These datasets all used post-mortem tissue from animals which died of causes unrelated to research, and therefore do not require a Home Office license under the Animals (Scientific Procedures) Act 1986. Ethics statements associated with all remaining Digital Brain Bank datasets are described in the original manuscript associated with each dataset, as provided in Table 1.

\section{Data Availability Statement}

The Digital Brain Bank (https://open.win.ox.ac.uk/DigitalBrainBank) is an interactive data discovery and release platform providing open access to curated, multimodal post-mortem neuroimaging datasets. All datasets described in this manuscript are available through the Digital Brain Bank, with details of access provided within the manuscript and on the website. Code for the Digital Brain Bank resource is available at https://git.fmrib.ox.ac.uk/thanayik/dbb. When available, details of associated processing code for each 
dataset is linked to the dataset's Information page on the Digital Brain Bank website. Source data for the corpus callosum analysis in Fig $3 \mathrm{c}$ is provided in a Supplementary File.

\section{Acknowledgements}

The Digital Brain Bank is supported by the Wellcome Trust (202788/Z/16/Z) and Medical Research Council (MRC, MR/K02213X/1). The Wellcome Centre for Integrative Neuroimaging is supported by core funding from the Wellcome Trust (203139/Z/16/Z).

KLM, BCT, AS and JM are supported by funding from the Wellcome Trust (202788/Z/16/Z), RBM is supported by funding from the Biotechnology and Biological Sciences Research Council (BBSRC) UK (BB/N019814/1) and the Netherlands Organization for Scientific Research NWO (452-13-015), SJ is supported by funding from the Wellcome Trust (221933/Z/20/Z, 215573/Z/19/Z) and the MRC (MR/L009013/1), TH and DM are supported by funding from the Wellcome Centre for Integrative Neuroimaging, $\mathbf{O A}$ is supported by funding from the Medical Research Council, Alzheimer's UK and NIHR Oxford Biomedical Research Centre, MFB is supported by funding from the Alfred Benzon's Foundation, KLB is supported by funding from the Biotechnology and Biological Sciences Research Council (BBSRC) UK (BB/N019814/1), SF and MPG are supported by funding from the MRC (MR/K02213X/1), MPvdH is supported by the Netherlands Organization for Scientific Research NWO (VIDI-45216-015) and (ALW-179), AFDH and IH are supported by funding from the Engineering and Physical Sciences Research Council (EPSRC, EP/L016052/1) and Medical Research Council (MRC, grant MR/L009013/1), AAK was funded by Cancer Research UK (grant C5255/A15935), PRM is supported by funding from the National Research Foundation of South Africa, RALM is supported by funding from the Medical Research Council (MR/K01014X/1) and the Wellcome Trust (202788/Z/16/Z), LR is supported by funding from the Biotechnology and Biological Sciences Research Council (BBSRC) UK (BB/M011224/1), JS is supported by funding from the IDEXLYON IMPULSION 2020 (IDEX/IMP/2020/14) and Labex CORTEX (ANR-11-LABX-0042) grant (Université de Lyon), CS is supported by funding from the NIHR Oxford Biomedical Research Centre (BRC), MRT is supported by funding from the Motor Neurone Disease Association, $\mathbf{C W}$ is supported by funding from the China Scholarship Council (CSC).

Human post-mortem brain datasets for the Digital Anatomist and Digital Pathologist used tissue provided by the Oxford Brain Bank, a research ethics committee (REC) approved, HTA regulated research tissue bank (REC reference 15/SC/0639). The Oxford Brain Bank is supported by the MRC, Brains for Dementia Research (BDR) (Alzheimer Society and Alzheimer Research UK), and the NIHR Oxford Biomedical Research Centre. The views expressed are those of the authors and not necessarily those of the NHS, the NIHR or the Department of Health. Datasets for the Digital Brain Zoo used tissue provided by the Australian Museum, Copenhagen Zoo, Primate Brain Bank, Save the Tasmanian Devil, Smithsonian, University of Oxford, and Zoological Society of London.

\section{Contributions}

Benjamin C. Tendler leads the Digital Brain Bank project, developed processing pipelines for Digital Anatomist (Human High-Resolution Diffusion MRI-PLI), Digital Brain Zoo (Hamadryas baboon, Gorilla, Chimpanzee and European Wolf), and Digital Pathologist (Human ALS MRI-Histology) MRI datasets, contributed to the establishment of MRI acquisition protocols for Digital Brain Zoo (Baboon and European Wolf) datasets, established the Digital Brain Bank website text and figures, contributed to Digital Brain Bank website design, and wrote the manuscript.

Taylor Hanayik implemented the Digital Brain Bank website, designed and implemented Tview, designed and implemented the website download/upload framework, contributed to the Digital Brain Bank website design and drafted sections of the manuscript.

Olaf Ansorge contributed to the development the of tissue sampling strategy, neuropathological analysis and recruitment of post-mortem brains tissue for Digital Anatomist (Human High-Resolution Diffusion MRI-PLI, Human Callosum MRI-PLI-Histology) and Digital Pathologist (Human ALS MRI-Histology) datasets.

Sarah Bangerter-Christensen carried out immunohistochemistry and histology image acquisition that generated the Human ALS MRI-Histology (Digital Pathologist) hippocampus histology dataset. 
Gregory S. Berns led the projects which acquired the Marsupial (Tasmanian Devil and Thylacine) and Cetacean (Common and Pantropical Dolphin) datasets (Digital Brain Zoo).

Mads F. Bertelsen acquired Carnivora (European Wolf) and Non-Human Primate (Hamadryas baboon, Cottontopped tamarin, Golden lion tamarin and Ring-tailed lemur) samples (Digital Brain Zoo).

Katherine L. Bryant led the project which acquired Primate Brain Bank (Bushbaby, Capuchin monkey, Chimpanzee, Colobus monkey, Mangabey, Night monkey, Saki monkey, Woolly monkey) datasets.

Sean Foxley conceived study design of the Human High-Resolution Diffusion MRI-PLI (Digital Anatomist) dataset, contributed to the establishment of MRI protocols and carried out MRI acquisition for the Human High-Resolution Diffusion MRI-PLI (Digital Anatomist), Non-Human Primate (Gorilla and Chimpanzee) (Digital Brain Zoo), and Human ALS MRI-Histology (Digital Pathologist) datasets, performed the tractography-PLI analysis for the Human High-Resolution Diffusion MRI-PLI (Digital Anatomist) dataset (Figs. $1 \mathrm{~b}$ and c), and drafted sections of the manuscript.

Martijn P. van den Heuvel contributed to acquisition of resources for the Digital Brain Zoo through the Primate Brain Bank (Bushbaby, Capuchin monkey, Chimpanzee, Colobus monkey, Mangabey, Night monkey, Saki monkey, Woolly monkey).

Amy F.D. Howard produced Tview images for the Digital Brain Bank website implementation, provided critical assessment of the Digital Brain Bank website, and contributed to Digital Brain Bank website design.

Istvan Huszar established the MRI-microscopy coregistration software (TIRL) and performed the MRI-histology coregistrations for the Human ALS MRI-Histology dataset (Digital Pathologist).

Alexandre A. Khrapitchev established the MRI acquisition protocols and carried out MRI acquisition for the small Non-Human Primate (Bushbaby, Capuchin monkey, Colobus monkey, Cotton-topped tamarin, Golden lion tamarin, Macaque monkey, Mangabey, Night monkey, Ring-tailed lemur, Saki monkey, Woolly monkey) datasets (Digital Brain Zoo).

Anna Leonte carried out immunohistochemistry and image acquisition for the Human ALS MRI-Histology (Digital Pathologist) anterior cingulate histology dataset.

Paul R. Manger acquired Carnivora (European Wolf) and Non-Human Primate (Hamadryas baboon, Cottontopped tamarin, Golden lion tamarin, Ring-tailed lemur) samples (Digital Brain Zoo).

Ricarda A.L. Menke provided critical assessment of the processing pipelines for Human ALS MRI-Histology dataset (Digital Pathologist).

Jeroen Mollink led the project which acquired the Human Callosum MRI-PLI-Histology (Digital Anatomist) dataset, and performed the PLI acquisition and analysis for the Human High-Resolution Diffusion MRI-PLI (Digital Anatomist) dataset.

Duncan Mortimer contributed to the Digital Brain Bank website design and established the data server implementation.

Menuka Pallebage-Gamarallage led the project which obtained the Human ALS MRI-Histology (Digital Pathologist) histology dataset, establishing protocols and carrying out systematic human brain sampling, immunohistochemistry, image acquisition and analyses of histology datasets. Supported Digital Anatomist projects (Human High-Resolution Diffusion MRI-PLI, Human Callosum MRI-PLI-Histology) with tissue sampling and histology data generation.

Lea Roumazeilles led projects which acquired Non-Human Primate (Chimpanzee, Gorilla, Macaque monkey, Ring-tailed lemur) datasets and facilitated the scanning preparation for Digital Brain Zoo samples scanned at the University of Oxford.

Jerome Sallet facilitated the acquisition and scanning preparation for all Digital Brain Zoo samples scanned at the University of Oxford.

Lianne H. Scholtens selected and prepared samples from the Primate Brain Bank (Bushbaby, Capuchin monkey, Chimpanzee, Colobus monkey, Mangabey, Night monkey, Saki monkey, Woolly monkey) (Digital Brain Zoo).

Connor Scott supported the recruitment and preparation of human whole brain samples for the Human HighResolution Diffusion MRI-PLI (Digital Anatomist) and Human ALS MRI-Histology (Digital Pathologist) datasets.

Adele Smart carried out immunohistochemistry and image acquisition for the Human ALS MRI-Histology (Digital Pathologist) histology dataset. 
Martin R. Turner supported the characterisation of the ALS cohort in the Human ALS MRI-Histology (Digital Pathologist) dataset.

Chaoyue Wang established the analysis pipeline and performed data processing of the T2* and magnetic susceptibility maps for the Human ALS MRI-Histology (Digital Pathologist) dataset.

Saad Jbabdi established analysis tools for processing diffusion MRI data in the Digital Anatomist (Human HighResolution Diffusion MRI-PLI), Digital Brain Zoo (Baboon, Chimpanzee, Gorilla and European Wolf), and Digital Pathologist (Human ALS MRI-Histology) datasets, contributed to the Digital Brain Bank website design, and critically appraised the manuscript.

Rogier B. Mars leads the Digital Brain Zoo, led projects associated with the first data release in the Digital Brain Zoo performed at the University of Oxford, contributed to the Digital Brain Bank website design, and critically appraised the manuscript.

Karla L. Miller conceived the Digital Brain Bank, led projects associated with the first data release in the Digital Anatomist and Digital Pathologist, contributed to the establishment of MRI acquisition protocols for Digital Anatomist (Human High-Resolution Diffusion MRI-PLI), Digital Brain Zoo (Hamadryas baboon, Chimpanzee, Gorilla and European Wolf), and Digital Pathologist (Human ALS MRI-Histology) datasets, contributed to the Digital Brain Bank website design, and critically appraised the manuscript.

All co-authors reviewed the manuscript 


\section{References}

Absinta, M., Ha, S.-K., Nair, G., Sati, P., Luciano, N. J., Palisoc, M., Louveau, A., Zaghloul, K. A., Pittaluga, S., Kipnis, J., \& others. (2017). Human and nonhuman primate meninges harbor lymphatic vessels that can be visualized noninvasively by MRI. Elife, 6, e29738.

Andersson, J. L. R., Jenkinson, M., \& Smith, S. (2007). Non-linear registration, aka Spatial normalisation FMRIB technical report TR07JA2. FMRIB Analysis Group of the University of Oxford, 2(1), e21.

Axer, M., Amunts, K., Grässel, D., Palm, C., Dammers, J., Axer, H., Pietrzyk, U., \& Zilles, K. (2011). A novel approach to the human connectome: Ultra-high resolution mapping of fiber tracts in the brain. Neurolmage. https://doi.org/10.1016/j.neuroimage.2010.08.075

Beaujoin, J., Palomero-Gallagher, N., Boumezbeur, F., Axer, M., Bernard, J., Poupon, F., Schmitz, D., Mangin, J.F., \& Poupon, C. (2018). Post-mortem inference of the human hippocampal connectivity and microstructure using ultra-high field diffusion MRI at 11.7 T. Brain Structure and Function, 223(5), 21572179.

Behrens, T. E. J., Berg, H. J., Jbabdi, S., Rushworth, M. F. S., \& Woolrich, M. W. (2007). Probabilistic diffusion tractography with multiple fibre orientations: What can we gain? Neurolmage. https://doi.org/10.1016/j.neuroimage.2006.09.018

Berns, G. S., \& Ashwell, K. W. S. (2017). Reconstruction of the cortical maps of the Tasmanian tiger and comparison to the Tasmanian devil. PLOS ONE. https://doi.org/10.1371/journal.pone.0168993

Berns, G. S., Cook, P. F., Foxley, S., Jbabdi, S., Miller, K. L., \& Marino, L. (2015). Diffusion tensor imaging of dolphin brains reveals direct auditory pathway to temporal lobe. Proceedings of the Royal Society B: Biological Sciences. https://doi.org/10.1098/rspb.2015.1203

Bhagwandin, A., Haagensen, M., \& Manger, P. R. (2017). The brain of the black (Diceros bicornis) and white (Ceratotherium simum) African rhinoceroses: morphology and volumetrics from magnetic resonance imaging. Frontiers in Neuroanatomy, 11, 74.

Birkl, C., Langkammer, C., Golob-Schwarzl, N., Leoni, M., Haybaeck, J., Goessler, W., Fazekas, F., \& Ropele, S. (2016). Effects of formalin fixation and temperature on MR relaxation times in the human brain. NMR in Biomedicine. https://doi.org/10.1002/nbm.3477

Birkl, C., Soellradl, M., Toeglhofer, A. M., Krassnig, S., Leoni, M., Pirpamer, L., Vorauer, T., Krenn, H., Haybaeck, J., Fazekas, F., Ropele, S., \& Langkammer, C. (2018). Effects of concentration and vendor specific composition of formalin on postmortem MRI of the human brain. Magnetic Resonance in Medicine. https://doi.org/10.1002/mrm.26699

Boon, B. D. C., Pouwels, P. J. W., Jonkman, L. E., Keijzer, M. J., Preziosa, P., van de Berg, W. D. J., Geurts, J. J. G., Scheltens, P., Barkhof, F., Rozemuller, A. J. M., \& others. (2019). Can post-mortem MRI be used as a proxy for in vivo? A case study. Brain Communications, 1(1), fcz030.

Bryant, K., Ardesch, D. J., Roumazeilles, L., Scholtens, L. H., Khrapitchev, A. A., Tendler, B. C., Wu, W., Miller, K. L., Sallet, J., den Heuvel, M. P., \& others. (2021). Diffusion MRI data, sulcal anatomy, and tractography for eight species from the Primate Brain Bank. Brain Structure and Function.

Calabrese, E., Hickey, P., Hulette, C., Zhang, J., Parente, B., Lad, S. P., \& Johnson, G. A. (2015). Postmortem diffusion MRI of the human brainstem and thalamus for deep brain stimulator electrode localization. Human Brain Mapping, 36(8), 3167-3178.

Cottaar, M., Bastiani, M., Boddu, N., Glasser, M. F., Haber, S., Van Essen, D. C., Sotiropoulos, S. N., \& Jbabdi, S. (2021). Modelling white matter in gyral blades as a continuous vector field. Neurolmage, 227, 117693.

D'Arceuil, H., \& de Crespigny, A. (2007). The effects of brain tissue decomposition on diffusion tensor imaging and tractography. Neurolmage. https://doi.org/10.1016/j.neuroimage.2007.02.039

D’Arceuil, H. E., Westmoreland, S., \& de Crespigny, A. J. (2007). An approach to high resolution diffusion tensor imaging in fixed primate brain. Neurolmage. https://doi.org/10.1016/j.neuroimage.2006.12.028

Dawe, R. J., Bennett, D. A., Schneider, J. A., Vasireddi, S. K., \& Arfanakis, K. (2009). Postmortem MRI of human brain hemispheres: T 2 relaxation times during formaldehyde fixation. Magnetic Resonance in Medicine. https://doi.org/10.1002/mrm.21909

Denic, A., Macura, S. I., Mishra, P., Gamez, J. D., Rodriguez, M., \& Pirko, I. (2011). MRI in rodent models of brain disorders. Neurotherapeutics, 8(1), 3-18.

Dyrby, T. B., Baaré, W. F. C., Alexander, D. C., Jelsing, J., Garde, E., \& Søgaard, L. V. (2011). An ex vivo imaging pipeline for producing high-quality and high-resolution diffusion-weighted imaging datasets. Human Brain Mapping. https://doi.org/10.1002/hbm.21043

Edlow, B. L., Mareyam, A., Horn, A., Polimeni, J. R., Witzel, T., Tisdall, M. D., Augustinack, J. C., Stockmann, J. P., 
Diamond, B. R., Stevens, A., \& others. (2019). 7 Tesla MRI of the ex vivo human brain at 100 micron resolution. Scientific Data, 6(1), 1-10.

Eichert, N., Robinson, E. C., Bryant, K. L., Jbabdi, S., Jenkinson, M., Li, L., Krug, K., Watkins, K. E., \& Mars, R. B. (2020). Cross-species cortical alignment identifies different types of anatomical reorganization in the primate temporal lobe. Elife, 9, e53232.

Foxley, S., Jbabdi, S., Clare, S., Lam, W., Ansorge, O., Douaud, G., \& Miller, K. (2014). Improving diffusionweighted imaging of post-mortem human brains: SSFP at 7T. Neurolmage. https://doi.org/10.1016/j.neuroimage.2014.08.014

Foxley, S., Mollink, J., Jbabdi, S., Clare, S., Hernandez Fernandez, M., Scott, C., Ansorge, O., \& Miller, K. L. (2016). Validating tractography of high resolution post-mortem human brain at 7T with polarized light imaging. ISMRM 24th Annual Meeting Singapore.

Friedrich, P., Forkel, S. J., Amiez, C., Balsters, J. H., Coulon, O., Fan, L., Goulas, A., Hadj-Bouziane, F., Hecht, E. E., Heuer, K., \& others. (2021). Imaging evolution of the primate brain: the next frontier? Neurolmage, 117685.

Fritz, F. J., Sengupta, S., Harms, R. L., Tse, D. H., Poser, B. A., \& Roebroeck, A. (2019). Ultra-high resolution and multi-shell diffusion MRI of intact ex vivo human brains using kT-dSTEAM at 9.4T. Neurolmage. https://doi.org/10.1016/j.neuroimage.2019.116087

Grewal, J. S., Gloe, T., Hegedus, J., Bitterman, K., Billings, B. K., Chengetanai, S., Bentil, S., Wang, V. X., Ng, J. C., Tang, C. Y., Geletta, S., Wicinski, B., Bertelson, M., Tendler, B. C., Mars, R. B., Aguirre, G. K., Rusbridge, C., Hof, P. R., Sherwood, C. C., ... Spocter, M. A. (2020). Brain gyrification in wild and domestic canids: Has domestication changed the gyrification index in domestic dogs? Journal of Comparative Neurology. https://doi.org/10.1002/cne.24972

Heuer, K., Gulban, O. F., Bazin, P.-L., Osoianu, A., Valabregue, R., Santin, M., Herbin, M., \& Toro, R. (2019). Evolution of neocortical folding: A phylogenetic comparative analysis of MRI from 34 primate species. Cortex, 118, 275-291.

Hofer, S., \& Frahm, J. (2006). Topography of the human corpus callosum revisited-Comprehensive fiber tractography using diffusion tensor magnetic resonance imaging. Neurolmage. https://doi.org/10.1016/j.neuroimage.2006.05.044

Howard, A. F., Mollink, J., Kleinnijenhuis, M., Pallebage-Gamarallage, M., Bastiani, M., Cottaar, M., Miller, K. L., \& Jbabdi, S. (2019). Joint modelling of diffusion MRI and microscopy. Neurolmage. https://doi.org/10.1016/j.neuroimage.2019.116014

Howard, A., Jbabdi, S., Khrapitchev, A., Sallet, J., Daubney, G., Mollink, J., Scott, C., Sibson, N., \& Miller, K. (2019). The BigMac dataset: ultra-high angular resolution diffusion imaging and multi-contrast microscopy of a whole macaque brain. ISMRM 27th Annual Meeting.

Huszar, I. N., Pallebage-Gamarallage, M., Foxley, S., Tendler, B. C., Leonte, A., Hiemstra, M., Mollink, J., Smart, A., Bangerter-Christensen, S., Brooks, H., \& others. (2019). Tensor Image Registration Library: Automated Non-Linear Registration of Sparsely Sampled Histological Specimens to Post-Mortem MRI of the Whole Human Brain. BioRxiv, 849570.

Iglesias, J. E., Modat, M., Peter, L., Stevens, A., Annunziata, R., Vercauteren, T., Lein, E., Fischl, B., Ourselin, S., Initiative, A. D. N., \& others. (2018). Joint registration and synthesis using a probabilistic model for alignment of MRI and histological sections. Medical Image Analysis, 50, 127-144.

Jenkinson, M., Beckmann, C. F., Behrens, T. E. J., Woolrich, M. W., \& Smith, S. M. (2012). FSL - Review. Neurolmage. https://doi.org/10.1016/j.neuroimage.2011.09.015

Jucker, M., \& Walker, L. C. (2013). Self-propagation of pathogenic protein aggregates in neurodegenerative diseases. Nature, 501(7465), 45-51.

Kaiser, R., Bartholdi, E., \& Ernst, R. R. (1974). Diffusion and field-gradient effects in NMR Fourier spectroscopy. The Journal of Chemical Physics. https://doi.org/10.1063/1.1681477

Kamman, R. L., Go, K. G., Stomp, G. P., Hulstaert, C. E., \& Berendsen, H. J. C. (1985). Changes of relaxation times T1 and T2 in rat tissues after biopsy and fixation. Magnetic Resonance Imaging. https://doi.org/10.1016/0730-725X(85)90353-4

Kassubek, J., Müller, H.-P., Del Tredici, K., Brettschneider, J., Pinkhardt, E. H., Lule, D., Böhm, S., Braak, H., \& Ludolph, A. C. (2014). Diffusion tensor imaging analysis of sequential spreading of disease in amyotrophic lateral sclerosis confirms patterns of TDP-43 pathology. Brain, 137(6), 1733-1740.

Keren, N. I., Taheri, S., Vazey, E. M., Morgan, P. S., Granholm, A.-C. E., Aston-Jones, G. S., \& Eckert, M. A. (2015). Histologic validation of locus coeruleus MRI contrast in post-mortem tissue. Neuroimage, 113, 235-245.

Klink, P. C., Aubry, J.-F., Ferrera, V. P., Fox, A. S., Froudist-Walsh, S., Jarraya, B., Konofagou, E., Krauzlis, R., 
Messinger, A., Mitchell, A. S., \& others. (2021). Combining Brain Perturbation and Neuroimaging in Nonhuman Primates. Neurolmage, 118017.

Langkammer, C., Schweser, F., Krebs, N., Deistung, A., Goessler, W., Scheurer, E., Sommer, K., Reishofer, G., Yen, K., Fazekas, F., Ropele, S., \& Reichenbach, J. R. (2012). Quantitative susceptibility mapping (QSM) as a means to measure brain iron? A post mortem validation study. Neurolmage. https://doi.org/10.1016/j.neuroimage.2012.05.049

Le Bihan, D. (1988). Intravoxel incoherent motion imaging using steady-state free precession. Magnetic Resonance in Medicine. https://doi.org/10.1002/mrm.1910070312

Liu, C. (2010). Susceptibility tensor imaging. Magnetic Resonance in Medicine, 63(6), 1471-1477. https://doi.org/10.1002/mrm.22482

Marcus, D. S., Wang, T. H., Parker, J., Csernansky, J. G., Morris, J. C., \& Buckner, R. L. (2007). Open Access Series of Imaging Studies (OASIS): cross-sectional MRI data in young, middle aged, nondemented, and demented older adults. Journal of Cognitive Neuroscience, 19(9), 1498-1507.

Mars, R. B., Jbabdi, S., \& Rushworth, M. F. S. (2021). A common space approach to comparative neuroscience. Annual Review of Neuroscience, 44.

Mars, R. B., Neubert, F.-X., Verhagen, L., Sallet, J., Miller, K. L., Dunbar, R. I. M., \& Barton, R. A. (2014). Primate comparative neuroscience using magnetic resonance imaging: promises and challenges. Frontiers in Neuroscience, 8, 298.

Mars, R. B., Sotiropoulos, S. N., Passingham, R. E., Sallet, J., Verhagen, L., Khrapitchev, A. A., Sibson, N., \& Jbabdi, S. (2018). Whole brain comparative anatomy using connectivity blueprints. Elife, 7, e35237.

Martins-Bach, A. B., Tachrount, M., Tisca, C., Qiu, L., Spring, S., Ellegood, J., Nieman, B. J., Sled, J. G., RaghavanNair, R., Fisher, E., Thomas, Cunningham, Lerch, J., \& L, M. K. (2021). Anatomical and microstructural brain alterations in the TDP-M323K mouse model of amyotrophic lateral sclerosis. International Society of Magnetic Resonance in Medicine, 1208.

McNab, J. A., Jbabdi, S., Deoni, S. C. L., Douaud, G., Behrens, T. E. J., \& Miller, K. L. (2009). High resolution diffusion-weighted imaging in fixed human brain using diffusion-weighted steady state free precession. Neurolmage. https://doi.org/10.1016/j.neuroimage.2009.01.008

McNab, J. A., \& Miller, K. L. (2010). Steady-state diffusion-weighted imaging: Theory, acquisition and analysis. In NMR in Biomedicine. https://doi.org/10.1002/nbm.1509

Merboldt, K. -D, Bruhn, H., Frahm, J., Gyngell, M. L., Hänicke, W., \& Deimling, M. (1989). MRI of "diffusion" in the human brain: New results using a modified CE-FAST sequence. Magnetic Resonance in Medicine. https://doi.org/10.1002/mrm.1910090316

Merboldt, K. D., Hxnicke, W., Gyngell, M. L., Frahm, J., \& Bruhn, H. (1989). Rapid NMR imaging of molecular self-diffusion using a modified CE-FAST sequence. Journal of Magnetic Resonance (1969). https://doi.org/10.1016/0022-2364(89)90170-4

Milham, M. P., Ai, L., Koo, B., Xu, T., Amiez, C., Balezeau, F., Baxter, M. G., Blezer, E. L. A., Brochier, T., Chen, A., \& others. (2018). An open resource for non-human primate imaging. Neuron, 100(1), 61-74.

Miller, K. L., Alfaro-Almagro, F., Bangerter, N. K., Thomas, D. L., Yacoub, E., Xu, J., Bartsch, A. J., Jbabdi, S., Sotiropoulos, S. N., Andersson, J. L. R., \& others. (2016). Multimodal population brain imaging in the UK Biobank prospective epidemiological study. Nature Neuroscience, 19(11), 1523-1536.

Miller, K. L., McNab, J. A., Jbabdi, S., \& Douaud, G. (2012). Diffusion tractography of post-mortem human brains: Optimization and comparison of spin echo and steady-state free precession techniques. Neurolmage. https://doi.org/10.1016/j.neuroimage.2011.09.054

Miller, K. L., Stagg, C. J., Douaud, G., Jbabdi, S., Smith, S. M., Behrens, T. E. J., Jenkinson, M., Chance, S. A., Esiri, M. M., Voets, N. L., Jenkinson, N., Aziz, T. Z., Turner, M. R., Johansen-Berg, H., \& McNab, J. A. (2011). Diffusion imaging of whole, post-mortem human brains on a clinical MRI scanner. Neurolmage. https://doi.org/10.1016/j.neuroimage.2011.03.070

Mollink, J., Kleinnijenhuis, M., Cappellen van Walsum, A. M. van, Sotiropoulos, S. N., Cottaar, M., Mirfin, C., Heinrich, M. P., Jenkinson, M., Pallebage-Gamarallage, M., Ansorge, O., Jbabdi, S., \& Miller, K. L. (2017). Evaluating fibre orientation dispersion in white matter: Comparison of diffusion MRI, histology and polarized light imaging. Neurolmage. https://doi.org/10.1016/j.neuroimage.2017.06.001

Nagara, H., Inoue, T., Koga, T., Kitaguchi, T., Tateishi, J., \& Goto, I. (1987). Formalin fixed brains are useful for magnetic resonance imaging (MRI) study. Journal of the Neurological Sciences. https://doi.org/10.1016/0022-510X(87)90184-5

Ohnishi, T., Nakamura, Y., Tanaka, T., Tanaka, T., Hashimoto, N., Haneishi, H., Batchelor, T. T., Gerstner, E. R., Taylor, J. W., Snuderl, M., \& others. (2016). Deformable image registration between pathological images and MR image via an optical macro image. Pathology-Research and Practice, 212(10), 927-936. 
Pallebage-Gamarallage, M., Foxley, S., Menke, R. A. L., Huszar, I. N., Jenkinson, M., Tendler, B. C., Wang, C., Jbabdi, S., Turner, M. R., Miller, K. L., \& Ansorge, O. (2018). Dissecting the pathobiology of altered MRI signal in amyotrophic lateral sclerosis: A post mortem whole brain sampling strategy for the integration of ultra-high-field MRI and quantitative neuropathology. BMC Neuroscience, 19(1). https://doi.org/10.1186/s12868-018-0416-1

Pfefferbaum, A., Sullivan, E. V., Adalsteinsson, E., Garrick, T., \& Harper, C. (2004). Postmortem MR imaging of formalin-fixed human brain. Neurolmage. https://doi.org/10.1016/j.neuroimage.2003.11.024

Roebroeck, A., Miller, K. L., \& Aggarwal, M. (2019). Ex vivo diffusion MRI of the human brain: Technical challenges and recent advances. NMR in Biomedicine. https://doi.org/10.1002/nbm.3941

Roumazeilles, L., Eichert, N., Bryant, K. L., Folloni, D., Sallet, J., Vijayakumar, S., Foxley, S., Tendler, B. C., Jbabdi, S., Reveley, C., Verhagen, L., Dershowitz, L. B., Guthrie, M., Flach, E., Miller, K. L., \& Mars, R. B. (2020). Longitudinal connections and the organization of the temporal cortex in macaques, great apes, and humans. PLoS Biology. https://doi.org/10.1371/journal.pbio.3000810

Roumazeilles, L., Lange, F. J., Benn, R. A., Andersson, J. L. R., Bertelsen, M. F., Manger, P. R., Flach, E., Khrapitchev, A. A., Bryant, K. L., Sallet, J., \& Mars, R. B. (2021). Cortical morphology and white matter tractography of three phylogenetically distant primates: Evidence for a simian elaboration. Cerebral Cortex.

Schilling, K., Gao, Y., Janve, V., Stepniewska, I., Landman, B. A., \& Anderson, A. W. (2018). Confirmation of a gyral bias in diffusion MRI fiber tractography. Human Brain Mapping. https://doi.org/10.1002/hbm.23936

Shepherd, T. M., Thelwall, P. E., Stanisz, G. J., \& Blackband, S. J. (2009). Aldehyde fixative solutions alter the water relaxation and diffusion properties of nervous tissue. Magnetic Resonance in Medicine. https://doi.org/10.1002/mrm.21977

Snoek, L., van der Miesen, M. M., Beemsterboer, T., Van Der Leij, A., Eigenhuis, A., \& Scholte, H. S. (2021). The Amsterdam Open MRI Collection, a set of multimodal MRI datasets for individual difference analyses. Scientific Data, 8(1), 1-23.

Sun, S. W., Neil, J. J., Liang, H. F., He, Y. Y., Schmidt, R. E., Hsu, C. Y., \& Song, S. K. (2005). Formalin fixation alters water diffusion coefficient magnitude but not anisotropy in infarcted brain. Magnetic Resonance in Medicine. https://doi.org/10.1002/mrm.20488

Sun, S. W., Neil, J. J., \& Song, S. K. (2003). Relative indices of water diffusion anisotropy are equivalent in live and formalin-fixed mouse brains. Magnetic Resonance in Medicine. https://doi.org/10.1002/mrm.10605

Tendler, B. C., Foxley, S., Cottaar, M., Jbabdi, S., \& Miller, K. L. (2020). Modeling an equivalent b-value in diffusion-weighted steady-state free precession. Magnetic Resonance in Medicine. https://doi.org/10.1002/mrm.28169

Tendler, B. C., Foxley, S., Hernandez-Fernandez, M., Cottaar, M., Scott, C., Ansorge, O., Miller, K. L., \& Jbabdi, S. (2020). Use of multi-flip angle measurements to account for transmit inhomogeneity and non-Gaussian diffusion in DW-SSFP. Neurolmage. https://doi.org/10.1016/j.neuroimage.2020.117113

Tendler, B. C., Qi, F., Foxley, S., Pallebage-Gamarallage, M., Menke, R. A. L., Ansorge, O., Hurley, S. A., \& Miller, K. L. (2020). A method to remove the influence of fixative concentration on post-mortem T2 maps using a Kinetic Tensor model. In bioRxiv. https://doi.org/10.1101/2020.09.16.299784

Thelwall, P. E., Shepherd, T. M., Stanisz, G. J., \& Blackband, S. J. (2006). Effects of temperature and aldehyde fixation on tissue water diffusion properties, studied in an erythrocyte ghost tissue model. Magnetic Resonance in Medicine. https://doi.org/10.1002/mrm.20962

Thiessen, J. D., Zhang, Y., Zhang, H., Wang, L., Buist, R., Del Bigio, M. R., Kong, J., Li, X.-M., \& Martin, M. (2013). Quantitative MRI and ultrastructural examination of the cuprizone mouse model of demyelination. NMR in Biomedicine, 26(11), 1562-1581.

Van Essen, D. C., Smith, S. M., Barch, D. M., Behrens, T. E. J., Yacoub, E., Ugurbil, K., Consortium, W.-M. H. C. P., \& others. (2013). The WU-Minn human connectome project: an overview. Neuroimage, 80, 62-79.

Vasung, L., Rezayev, A., Yun, H. J., Song, J. W., van der Kouwe, A., Stewart, N., Palani, A., Shiohama, T., Chouinard-Decorte, F., Levman, J., \& Takahashi, E. (2019). Structural and Diffusion MRI Analyses With Histological Observations in Patients With Lissencephaly. Frontiers in Cell and Developmental Biology. https://doi.org/10.3389/fcell.2019.00124

Wang, C., Foxley, S., Ansorge, O., Bangerter-Christensen, S., Chiew, M., Leonte, A., Menke, R. AL, Mollink, J., Pallebage-Gamarallage, M., Turner, M. R., Miller, K. L., \& Tendler, B. C. (2020). Methods for quantitative susceptibility and R2* mapping in whole post-mortem brains at 7T applied to amyotrophic lateral sclerosis. Neurolmage. https://doi.org/10.1016/j.neuroimage.2020.117216

Weigel, M. (2015). Extended phase graphs: Dephasing, RF pulses, and echoes - Pure and simple. In Journal of 
bioRxiv preprint doi: https://doi.org/10.1101/2021.06.21.449154; this version posted September $1,2021$. The copyright holder for this

preprint (which was not certified by peer review) is the author/funder, who has granted bioRxiv a license to display the preprint in perpetuity. It is made available under aCC-BY 4.0 International license.

Magnetic Resonance Imaging. https://doi.org/10.1002/jmri.24619

Weigel, M., Dechent, P., Galbusera, R., Bahn, E., Nair, G., Kappos, L., Brück, W., Stadelmann, C., \& Granziera, C. (2021). Imaging Multiple Sclerosis Pathology at $160 \mu \mathrm{m}$ Isotropic Resolution by Human Whole-Brain Ex Vivo Magnetic Resonance Imaging at 3T. BioRxiv.

Wilhelm, M. J., Ong, H. H., Wehrli, S. L., Li, C., Tsai, P.-H., Hackney, D. B., \& Wehrli, F. W. (2012). Direct magnetic resonance detection of myelin and prospects for quantitative imaging of myelin density. Proceedings of the National Academy of Sciences, 109(24), 9605-9610.

Wilkinson, M., Wang, R., van der Kouwe, A., \& Takahashi, E. (2016). White and gray matter fiber pathways in autism spectrum disorder revealed by ex vivo diffusion MR tractography. Brain and Behavior. https://doi.org/10.1002/brb3.483

Wu, W., Baxter, L., Adams, E., Andritsou, F., Bastiani, M., Fry, R. E., Frost, R., Foxley, S., Gallagher, C., Moultrie, F., Monk, V., Porter, D. A., Price, A., Rieger, S. W., Sanders, M., Edwards, A. D., Hajnal, J. V, Slater, R., \& Miller, K. L. (2021). High resolution diffusion imaging in the unfixed post-mortem neonatal brain. International Society of Magnetic Resonance in Medicine, 2246.

Yong-Hing, C. J., Obenaus, A., Stryker, R., Tong, K., \& Sarty, G. E. (2005). Magnetic resonance imaging and mathematical modeling of progressive formalin fixation of the human brain. Magnetic Resonance in Medicine. https://doi.org/10.1002/mrm.20578 